\title{
The Role of Embodied Carbon Databases in the Accuracy of Life Cycle Assessment (LCA) Calculations for the Embodied Carbon of Buildings
}

\author{
Golnaz Mohebbi ${ }^{1}$, Ali Bahadori-Jahromi ${ }^{1, *}$, Marco Ferri ${ }^{2}$ and Anastasia Mylona ${ }^{3}$ \\ 1 Department of Civil Engineering and Built Environment, School of Computing and Engineering, \\ University of West London, London W5 5RF, UK; 21460515@student.uwl.ac.uk \\ 2 Lidl Great Britain Ltd., 19 Worple Road, London SW19 4JS, UK; marco.ferri@lidl.co.uk \\ 3 Research Department, The Chartered Institution of Building Services Engineers [CIBSE], \\ London SW12 9BS, UK; AMylona@cibse.org \\ * Correspondence: Ali.Bahadori-Jahromi@uwl.ac.uk
}

check for updates

Citation: Mohebbi, G.;

Bahadori-Jahromi, A.; Ferri, M.;

Mylona, A. The Role of Embodied

Carbon Databases in the Accuracy of

Life Cycle Assessment (LCA)

Calculations for the Embodied

Carbon of Buildings. Sustainability

2021, 13, 7988. https://doi.org/

$10.3390 /$ su13147988

Academic Editor: Marco Raugei

Received: 23 June 2021

Accepted: 13 July 2021

Published: 16 July 2021

Publisher's Note: MDPI stays neutral with regard to jurisdictional claims in published maps and institutional affiliations.

Copyright: (c) 2021 by the authors. Licensee MDPI, Basel, Switzerland. This article is an open access article distributed under the terms and conditions of the Creative Commons Attribution (CC BY) license (https:/ / creativecommons.org/licenses/by/ $4.0 /)$.

\begin{abstract}
Studies conducted by major national and international scientific bodies have indisputably concluded that the increase in anthropogenic greenhouse gas emissions (GHG) since the mid-20th century has led to irreversible changes in the climate. Data has shown that the contribution of the building sector accounts for 39\% of these emissions. Reducing GHG emissions associated with the construction phase of buildings, or embodied carbon (EC), will prevent GHG emissions from entering the atmosphere earlier, reducing the negative impacts. However, to achieve any meaningful reduction, there is a need for consistency and accuracy in the calculations. The accuracy of these calculations is primarily tied to the accuracy of embodied carbon factors (ECF) used in the calculations, values determining the environmental impact of a product or procedure per unit weight. The emissions of any product can be calculated by performing a Life Cycle Assessment (LCA). While the requirements for carrying out an LCA have been standardised in ISO14044, the lack of a definitive national ECF database in the UK means that EC calculations can vary drastically based on the chosen database. An LCA has been carried out on a standard Lidl supermarket design within the A1-A3 boundary. For the calculation, the ECFs were sourced from two different databases, using the GHG conversion factor data published in 2020 by the UK Department of Energy \& Climate Change and data published in 2019 by the Inventory of Carbon and Energy (ICE). The latter is currently accepted as the most consistent database for carbon factors in the UK. This study showed that using a more detailed database compared to using a more general database could result in a $35.2 \%$ reduction of embodied carbon, while using more detailed data from a single database can reduce it by a further $5.5 \%$. It is necessary to establish the most accurate baseline for embodied carbon so that any carbon reduction attempts can be as effective as possible.
\end{abstract}

Keywords: embodied carbon; LCA; embodied carbon factor; EC; ECF; BIM

\section{Introduction}

The reduction of greenhouse gas (GHG) emissions is a vital task that needs to be taken on a global level to mitigate the negative impacts of climate change. According to a 2019 report from the United Nations Environment Programme (UNEP), the building sector accounts for $39 \%$ of the global energy-based GHG emissions, which have increased by $6 \%$ in the past decade [1].

The building sector has a high potential for emission reduction [2]. However, the focus on the operational phase in the past [3] has neglected the $6 \%$ of all global energy use and the $11 \%$ of energy-based GHG emissions that are attributed to the construction industry [1]. The focus on the operational phase has resulted from several factors. The main factor has been the assumption that the operational phase contributes a more significant percentage of a building's lifetime emissions and energy use, up to $90 \%$ in a 50-year 
operational lifespan [4,5]. More recent studies have determined that, given the parameters of a case study, this ratio can expand so that the energy use and subsequent emissions during the operational phase can contribute as low as 32\% [6]. This focus on the operational phase has led to the development of various software and methodologies for calculating operational energy use, making the process easier, which, in turn, is another influencing factor. Another result of this focus is a shift in the ratio between the emissions during construction and emissions during the use phase towards the former, causing the construction period emissions to contribute a more significant portion to the lifetime emissions and the consequent negative impact of those emissions. A vital factor to consider is that the operational emissions are produced during a more extended period compared to construction emissions, which are produced during a shorter period and also at the beginning of a building's timeline. Consequently, the GHG emissions will enter the atmosphere earlier, and the negative impacts will be seen across an extended period.

These facts highlight the necessity to focus on the energy used and GHG emissions during the construction period. Regardless of the carbon reduction methodology, the accuracy of the starting carbon amount is vital in determining an accurate and achievable reduction goal. This study has observed how decisions during this step can result in inaccurate data. It has also surmised how inaccuracies in the calculations during this stage can hinder the carbon reduction process.

\subsection{Embodied Energy and Embodied Carbon}

Embodied energy is all energy used to produce a product, from the extraction of raw material, transport, and the production process. The emissions of a building can be divided into three sections. The first are the emissions produced during the construction up to practical completion. This includes the embodied carbon of the building materials, transport of those materials, and all emissions produced during the on-site construction [7]. The emissions produced on-site include the energy use of construction equipment, temporary on-site structures, material waste, and the emissions associated with on-site waste disposal [8]. Each greenhouse gas is given a global warming potential (GWP), and in order to make a comparison possible, all GHGs emitted are converted to their carbon dioxide equivalent, $\mathrm{CO} 2 \mathrm{e}$. In order to calculate the embodied carbon, all of the energy used during the production process is broken down by energy source and the GHG emissions produced by that type of energy source or fuel [9] which are then converted to the CO2 equivalent. Recurring embodied carbon is all the GHG emissions produced from the refurbishment and repairs to the building's structure [10]. Although these emissions occur further down the building's lifetime, they are still majorly determined by the factors contributing to the initial embodied carbon and need to be included when calculating the embodied carbon of buildings. The scope of the assessments carried out in some studies may or may not include the recurring embodied carbon. The length of the lifespan of buildings directly affects the level of recurring embodied carbon [11]. The second section of a building's emissions are the emissions produced during the use phase of a building. These include the carbon emitted for maintenance, the operational energy and water use, or the operational carbon. The third is all carbon emissions produced during the demolition and disposal of buildings [7]. This study has focused on the first section of the building's emissions or the embodied carbon of the building, not including the recurring embodied carbon.

\subsection{Embodied Carbon Calculations}

\subsubsection{LCA Methodology (A1-A3, A1-A5)}

A Life Cycle Assessment (LCA) is a tool that can be used to calculate embodied carbon. An LCA is a process in which the potential environmental impacts for a product throughout its lifecycle is calculated. In 1997, the International Organization for Standards (ISO) published ISO 14,040 to set the standards guideline for performing an LCA, which was later amended in 2006 and 2020 and published in ISO 14,044 [12-14]. A product's lifecycle is typically divided into four main stages: 1-Material manufacturing, 2-Construction, 
3-Use and maintenance, and 4-End of life (Figure 1). An LCA could include one or any number of these stages. The process of calculating the embodied carbon of a building can be divided into a cradle-to-gate LCA (A1-A3) and a cradle-to-cradle LCA which covers only one part of the life cycle, e.g., construction process (A4-A5) [15]. The process of performing an LCA is primarily done in four stages. 1. Goal and Scope: Determines which processes of the unit's life cycle will be included in the assessment. 2. Life Cycle Inventory (LCI): Collection of input data needed for the assessment. 3. Life Cycle Impact Assessment (LCIA): Evaluation of the size and significance of the environmental impacts of a product throughout its life cycle. 4. Life Cycle Interpretation: Analysis of the results of the LCI and LCIA within the goal and scope. This analysis can provide recommendations for the reduction of the environmental impact, relative to the goal and scope of the LCA. This stage can also include reviewing, revising, and refining the LCA methodology (Figure 2) [12].

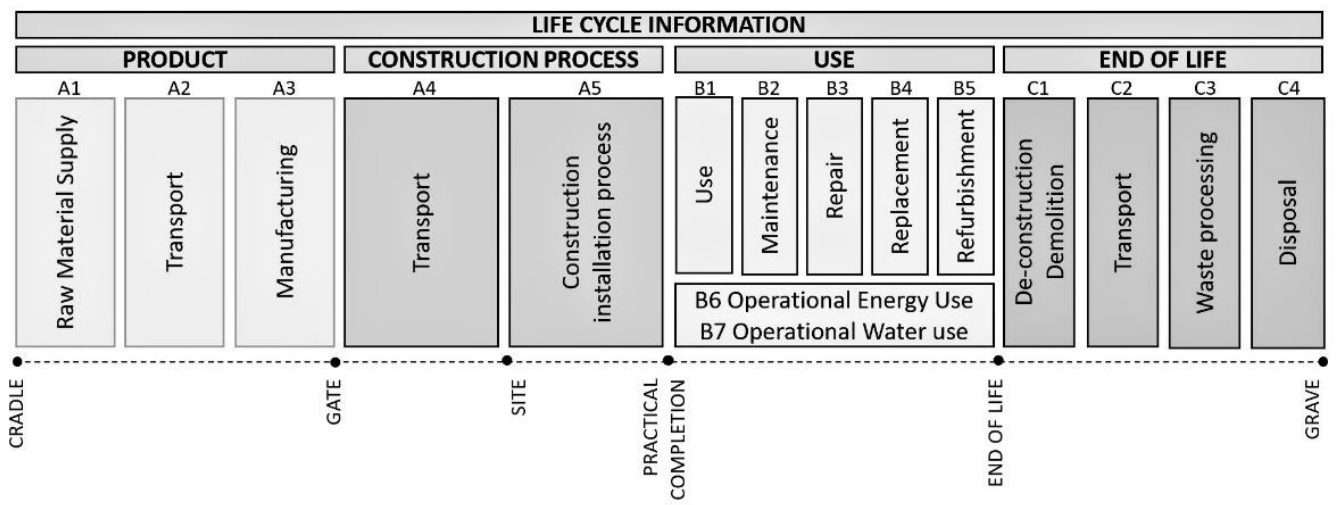

Figure 1. Life cycle stages reproduced from istructe How to calculate embodied carbon [11].

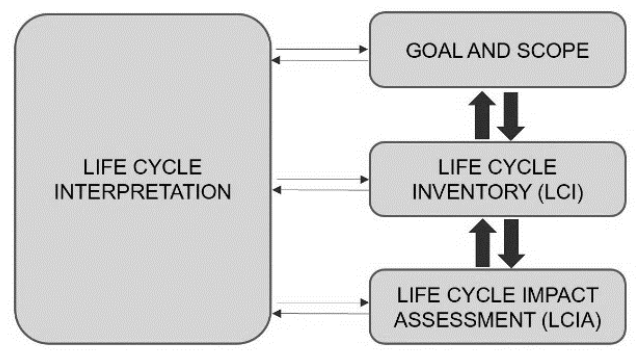

Figure 2. LCA stages reproduced from ISO 14,040 [14].

\subsubsection{A1-A3 Calculation}

When performing an LCA within the A1-A3 boundary, Equation (1) [8] can be used to calculate the embodied carbon of materials. There are two sets of input data required for this calculation, collected during the LCI stage.

Material quantity $(\mathrm{Kg}) \times$ Carbon factor $(\mathrm{KgCO} 2 \mathrm{e} / \mathrm{Kg})=$ Embodied Carbon $(\mathrm{KgCO} 2 \mathrm{e})$

The material weight can be calculated in one of two ways, dependent on at what stage of the construction the LCA is being performed. If the LCA is being carried out as a case study post-construction, a Process LCA (PLCA) (a process used when the physical flow of all aspects can be identified and traced [16]) approach can be taken to collect all data. If the LCA is performed pre-construction, material weights can be derived from Building Information Modelling (BIM). In the former scenario, the number of unknown elements is significantly reduced, such as material waste. In contrast, in the latter scenario, these elements must be assumed and do not provide adequate guidelines for carbon reduction in industry situations. Most LCAs have been performed as case studies of existing buildings. 
While adding valuable information to this area of knowledge, the post hoc nature of these studies does not provide the opportunity for early design intervention [3].

\subsubsection{Embodied Carbon Factor (ECF) Databases}

The second set of input data required for embodied carbon calculations are carbon factors. Carbon factors provide an estimation of the GWP impact of each product or process. Obtaining correct and accurate values are an essential part of performing an LCA. Carbon factors can be derived from several secondary sources in order to perform an assessment. These will include [17]:

- Environmental Product Declarations (EPD) [11];

- Industry data;

- Government data;

- Factor from commercial LCA database (ICE database);

- PAS 2050 compliant carbon footprint;

- Factor derived/aggregated from literature.

Each data source varies in detail, specificity, and accuracy. The most accurate data can be obtained from EPDs. EPDs are assessments carried out by manufacturers adhering to the requirements of BS EN 15804:2012 and A2:2019. The process in which the EPD is produced must also adhere to the ISO 14,044 standard. Based on EN 15,804 requirements, an EPD is only required to be performed within the A1-A3 boundary, and all other life cycle stages can be performed voluntarily. However, there is no requirement for manufacturers to publish EPDs, even though the number of published EPDs is steadily increasing (3500 globally in 2017 [18]), so the number of available EPDs are limited.

In cases where the other stages are included in the EPD, standard practices of the area or country where the EPD is published must be considered. For instance, a cradle-to-grave EPD published in Germany is more likely to assume energy recovery for the end-of-life procedure of a material. Meanwhile, in the UK, the end-of-life of materials is typically either recycling or landfill disposal [19]. Other sources for carbon data are industry data and national databases. In 1999, later amended in 2009, BRE published the Environmental Profiles Methodology highlighting the embodied carbon of over 200 of the most common building materials using data procured from the UK building industry. One of the most updated and widely used databases in the UK is the Inventory of Carbon and Energy (ICE) databases produced by Hammond and Jones in 2008. This database has been updated regularly, with the most recent update published in 2019 [20]. This database provides the cradle-to-gate carbon factor of over 500 of the most common building materials. This study investigates how the choice of ECF database and the approach taken for assigning an ECF to materials during an LCA can affect the outcome.

\section{Methodology}

For the purposes of this study, an A1-A3 LCA was carried out for a standard Lidl supermarket. The standard design for these supermarkets is a $2500 \mathrm{~m}^{2}$ single-story layout (Figure 3a-e). The structure of Lidl supermarkets in the UK is composed of a steel frame and composite external walls. The standard design for Lidl supermarket buildings was simulated using the BIM software Autodesk ${ }^{\circledR}$ Revit $^{\circledR}$ (Figure $4 a, b$ ) based on the design plans and data provided by Lidl GB Ltd. to calculate the weight for each of these materials and their composing components. To perform an A1-A3 LCA, the minimum elements that would need to be included are the substructure and the superstructure. The substructure consists of the foundation and the ground-bearing slabs of the building. The superstructure consists of the structural frame, upper floors, roof, stairs and ramps, and external and internal walls and partitions [8]. The details included in the BIM model were floors, structural foundation, roofs, structural columns and framing, walls, windows, and the mechanical duct system. The AHU (air handling units), electrical and plumbing systems were excluded from the model and calculations. The carbon contribution of these building components is not contained within the A1-A3 boundary as the calculation for these 
components must also include the recurring embodied carbon [11]. All materials used in the Lidl standard design in the UK are presented in Table 1 according to Lidl designs.

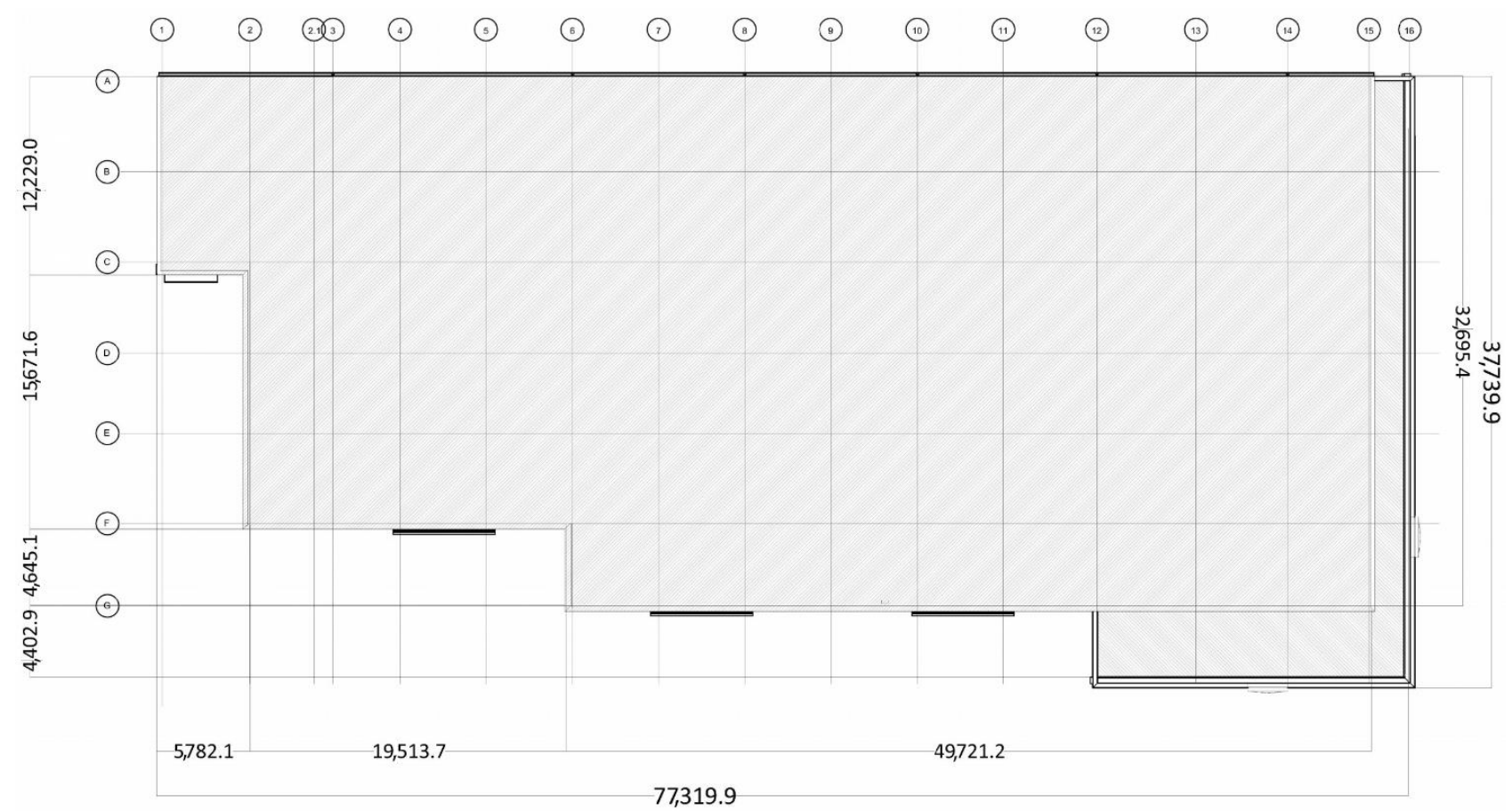

(a)

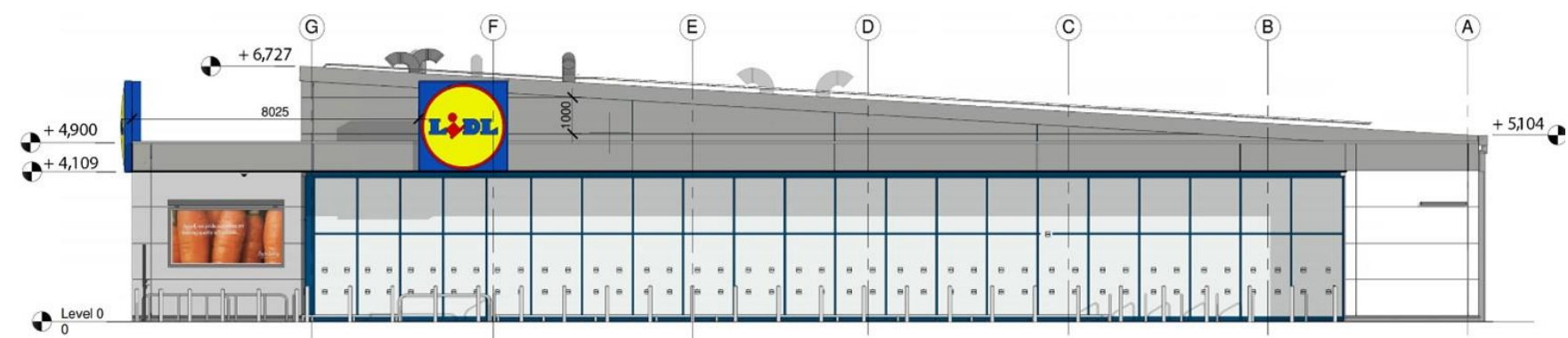

(b)

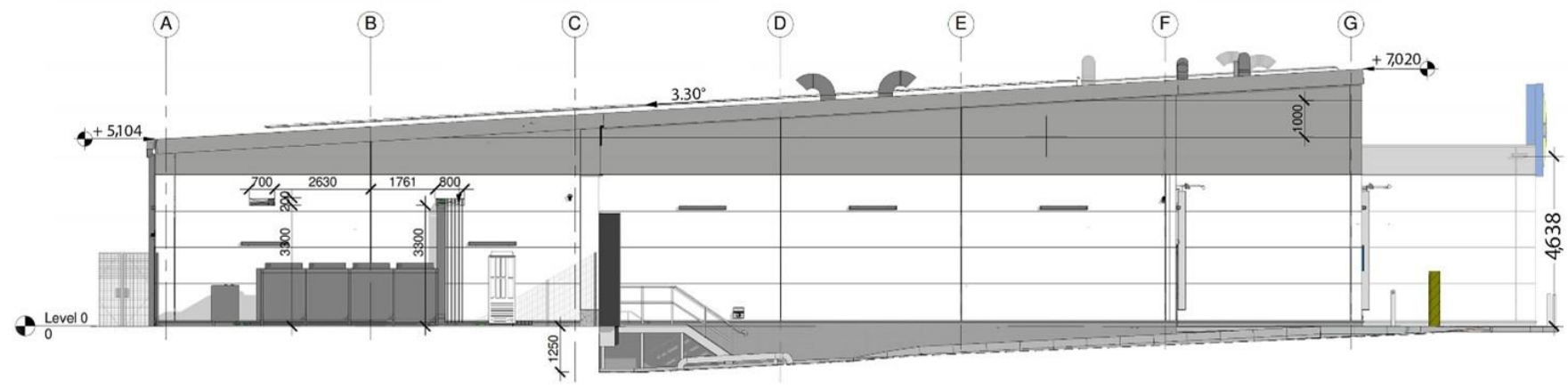

(c)

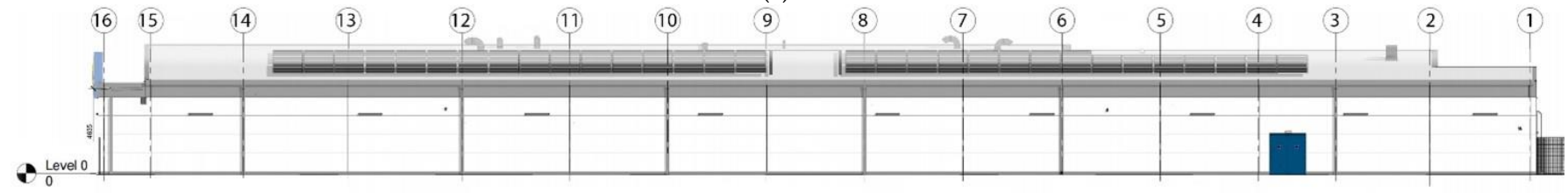

(d)

Figure 3. Cont. 


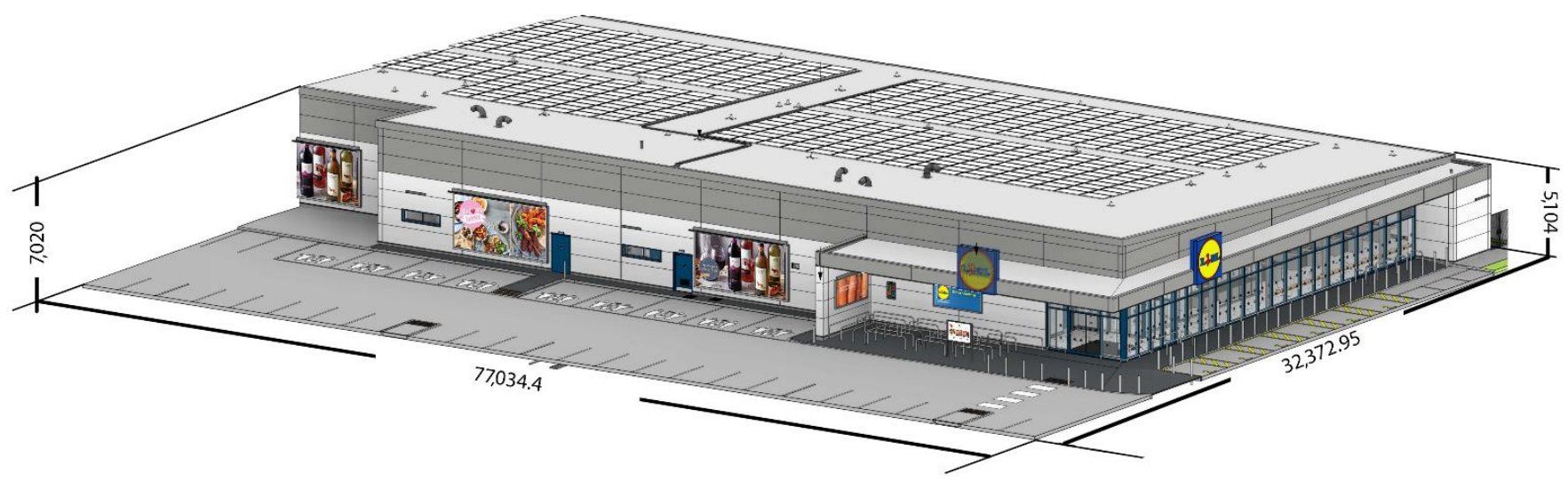

(e)

Figure 3. A 3D model and plans of Lidl standard design: (a) Building footprint; (b-d) Façades; (e) 3D model of Lidl standard supermarket design.

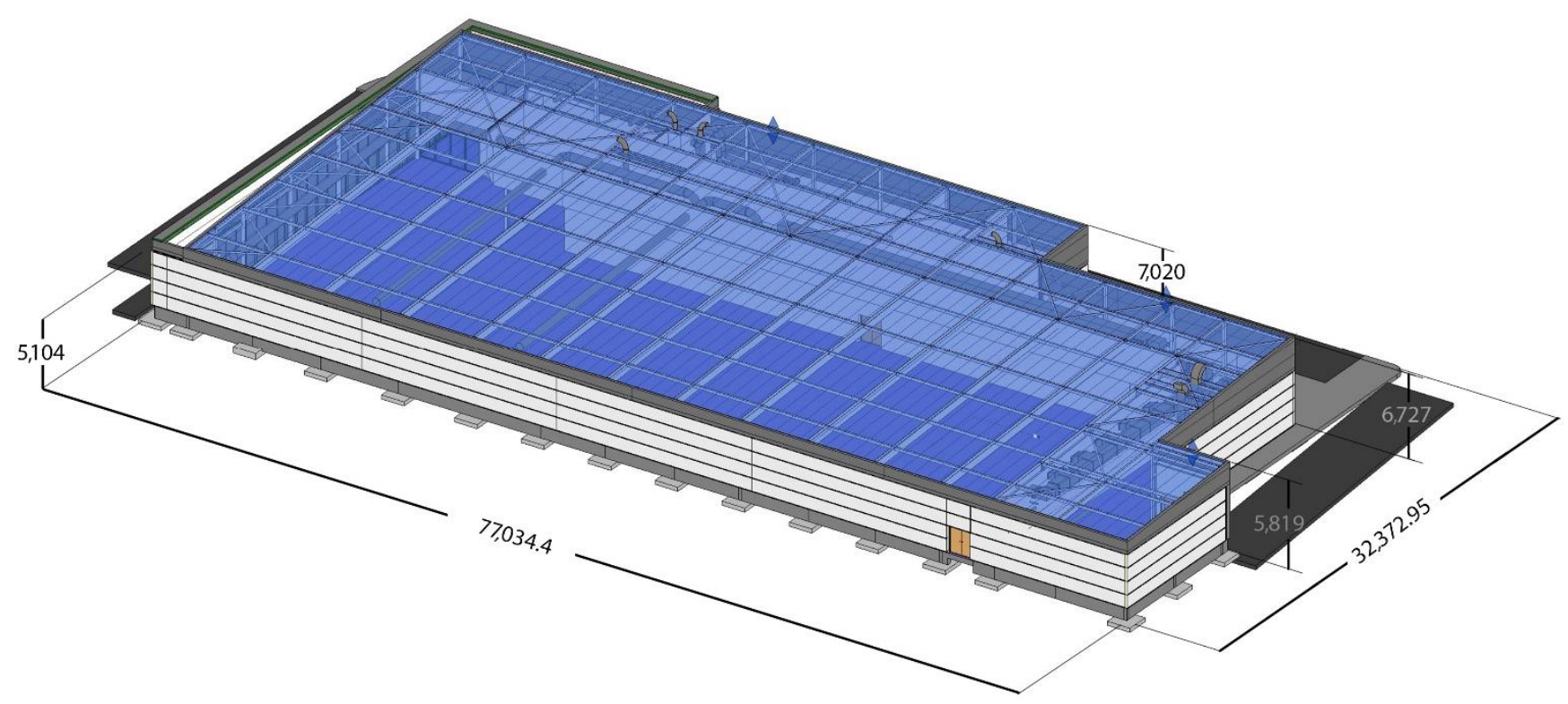

(a)

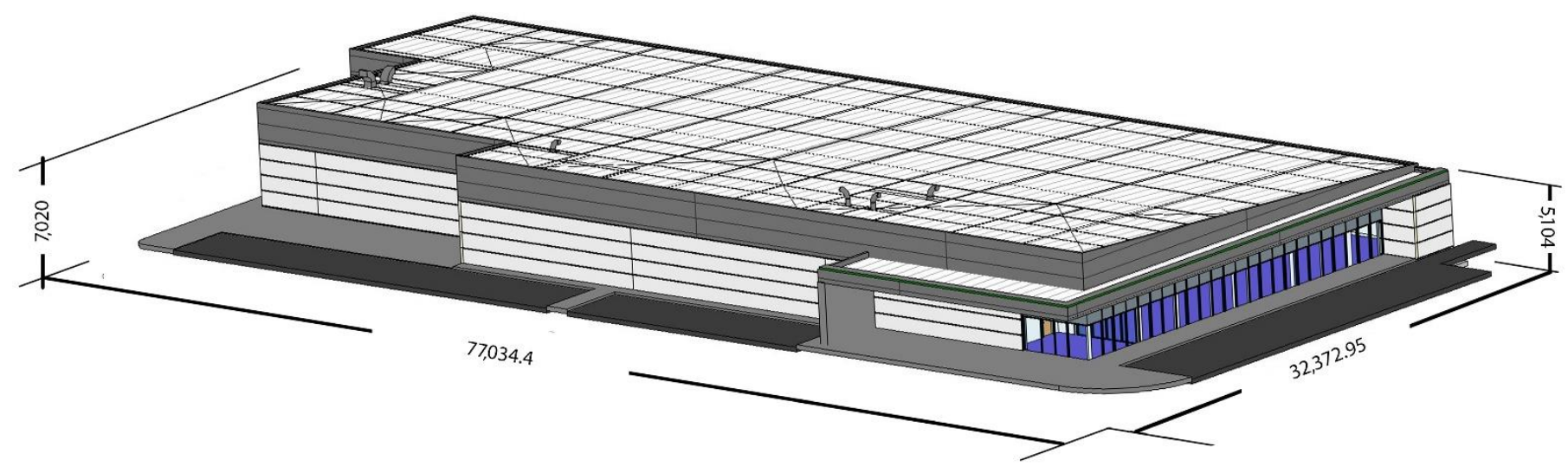

(b)

Figure 4. Simulated standard design model for Lidl supermarket buildings using Autodesk ${ }^{\circledR} \operatorname{Revit}^{\circledR}$ BIM software: $(\mathbf{a}, \mathbf{b})$ Isometric view of the model. 
Table 1. Material list of structural material used in Lidl standard design supermarkets in the UK.

\begin{tabular}{|c|c|c|c|}
\hline Building Element & Structural Element & Component & Component Details \\
\hline \multirow{4}{*}{ Substructure } & Floors & Floor & Floor: Floor-Upper $160 \mathrm{~mm}$ Concrete \\
\hline & Structural Foundations & Foundation Slab & Foundation Slab: Standard with tiles \\
\hline & Structural Foundations & Pile Cap & Pile Cap: $1500 \times 1500 \times 500$ \\
\hline & Structural Foundations & Wall Foundation & Wall Foundation: Standard \\
\hline \multirow[t]{2}{*}{ Envelope } & Roofs & Basic Roof & $\begin{array}{l}\text { Metal roof panels, flat soffit-NBS } \\
\qquad 20-50-55 / 150\end{array}$ \\
\hline & Roofs & Basic Roof & Metal single skin, $125 \mathrm{~mm}$ insulation \\
\hline \multirow{10}{*}{ Framing } & Structural Columns & Square Hollow Sections-Column & $\begin{array}{c}\text { RHS200 } \times 100 \times 6.3 \\
\text { SHS } 00 \times 80 \times 8 \\
\text { SHS150 } \times 150 \times 5 \\
\text { SHS200 } \times 200 \times 6.3 \\
\text { SHS250 } \times 250 \times 6.3\end{array}$ \\
\hline & Structural Columns & UC-Universal Columns-Column & $\begin{array}{l}\mathrm{UC} 152 \times 152 \times 23 \\
\mathrm{UC} 152 \times 152 \times 30 \\
\mathrm{UC} 203 \times 203 \times 46\end{array}$ \\
\hline & Structural Framing & Circular Hollow Sections & CHS114.3 × 5 \\
\hline & Structural Framing & $\begin{array}{l}\text { Light Gauge-Kingspan Multibeam } \\
\text { Purlins }\end{array}$ & M205065180 \\
\hline & Structural Framing & $\begin{array}{c}\text { Light Gauge-Kingspan Multibeam } \\
\text { Rails }\end{array}$ & M205065180 \\
\hline & Structural Framing & PFC-Parallel Flange Channels & $\begin{array}{l}\mathrm{PFC} 150 \times 75 \times 18 \\
\mathrm{PFC} 200 \times 75 \times 23\end{array}$ \\
\hline & Structural Framing & Plate & Plate: $10 \times 100$ PLT \\
\hline & Structural Framing & Rectangular Hollow Sections & $\begin{array}{l}\text { RHS140 × } 80 \times 6.3 \\
\text { RHS200 } \times 100 \times 6.3\end{array}$ \\
\hline & Structural Framing & Square Hollow Sections & $\begin{array}{c}\text { SHS150 } \times 150 \times 5 \\
\text { SHS180 } \times 180 \times 6.3\end{array}$ \\
\hline & Structural Framing & UB-Universal Beams & $\begin{array}{l}\text { UB203 } \times 133 \times 25 \\
\text { UB203 } \times 133 \times 25 \\
\text { UB254 } \times 146 \times 31 \\
\text { UB305 } \times 165 \times 54 \\
\text { UB406 } \times 140 \times 39 \\
\text { UB533 } \times 210 \times 122 \\
\text { UB610 } \times 229 \times 101 \\
\text { UB686 } \times 254 \times 125\end{array}$ \\
\hline \multirow{4}{*}{ Envelope } & Walls & External wall type 1 & $\begin{array}{l}\text { Longspan insulated cladding panels } \\
(1000 \mathrm{~mm}) \text { fixed to primary steel columns }\end{array}$ \\
\hline & Walls & External wall type 2 & $\begin{array}{l}365 \mathrm{~mm} \text { concrete wall ( } 80 \mathrm{~mm} \text { insulation) } \\
\text { faced with } 20 \mathrm{~mm} \text { render to external side, } \\
\text { painted white concrete face internally } \\
\text { (grey plinth at ground level externally) }\end{array}$ \\
\hline & Walls & External wall type 3 & $\begin{array}{c}\text { Canopy end wall }-2 \times 60 \mathrm{~mm} \text { Cladding } \\
\text { panels fixed back to Secondary steelwork } \\
\text { via top hats. Grey rendered plinth } \\
\text { externally at ground level }\end{array}$ \\
\hline & Walls & $\begin{array}{l}\text { Internal wall type } 1 \mathrm{~A}(300 \mathrm{~mm}) \\
\text { Internal wall type } 1 \mathrm{~B}(399 \mathrm{~mm}) \\
\text { Internal wall type } 1 \mathrm{C}(428 \mathrm{~mm})\end{array}$ & $\begin{array}{l}\text { Metal stud twin frame }+15 \mathrm{~mm} \text { Duraline } \\
\text { on each side }\end{array}$ \\
\hline
\end{tabular}


Table 1. Cont.

\begin{tabular}{|c|c|c|c|}
\hline Building Element & Structural Element & Component & Component Details \\
\hline & Walls & Internal wall type 2 & $\begin{array}{c}146 \mathrm{~mm} \text { metal stud }+15 \mathrm{~mm} \text { Duraline on } \\
\text { each side }\end{array}$ \\
\hline & Walls & Internal wall type 3 & $\begin{array}{l}70 \mathrm{~mm} \text { metal stud }+15 \mathrm{~mm} \text { Duraline on } \\
\text { each side }\end{array}$ \\
\hline & Walls & Internal wall type 4 & $\begin{array}{c}70 \mathrm{~mm} \text { metal stud }+15 \mathrm{~mm} \text { Duraline on } \\
\text { one side }\end{array}$ \\
\hline & Walls & Internal wall type 5 & $\begin{array}{l}92 \mathrm{~mm} \text { metal stud }+15 \mathrm{~mm} \text { Duraline on } \\
\text { one side }\end{array}$ \\
\hline & Walls & Internal wall type 6 & $\begin{array}{c}140 \mathrm{~mm} \text { blockwork }+15 \mathrm{~mm} \text { Plaster on } \\
\text { each side }\end{array}$ \\
\hline & Walls & Internal wall type 7 & $\begin{array}{l}140 \mathrm{~mm} \text { concrete }+15 \mathrm{~mm} \text { Plaster on } \\
\text { each side }\end{array}$ \\
\hline \multirow{3}{*}{ Curtain wall } & Windows & Curtain wall opening & $2100 \mathrm{~mm} \times 4000 \mathrm{~mm}$ clear opening \\
\hline & Windows & Curtain wall type 1 & $1430 \mathrm{~mm} \times 4000 \mathrm{~mm}$ curtain wall \\
\hline & Windows & Curtain wall type 2 & $\begin{array}{c}1050 \mathrm{~mm}-2050 \mathrm{~mm} \times 4000 \mathrm{~mm} \\
\text { curtain wall }\end{array}$ \\
\hline
\end{tabular}

\subsection{Revit ${ }^{\circledR}$ Building Information Modelling (BIM)}

The modelling of the building was initially carried out without assigning specific materials and using Revit ${ }^{\circledR}$ default materials for each building element. Each building element was then assigned the corresponding material according to Lidl plan specifications. The material assigned for each building element was selected based on the following order of preference:

- Manufacturer-provided BIM building material;

- Manual recreation of BIM building material according to manufacturer details;

- Manual recreation of BIM building material according to plan specifications;

- Generic/Default materials.

According to plan specifications, the material for the model's roof is a metal insulated sandwich panel roofing system. The BIM material published by Kingspan, hosted in the National BIM library, "Kingspan Insulated Panels-Metal Insulating Sandwich Panel Roofing System-Quad Core Trapezoidal Roof Panel-KS1000RW $120 \mathrm{~mm}$ ", was assigned to the main roof component. For the external wall type 1, a BIM material published by Arcelor Mittal, "Flamstyl S ArcelorMittal-1000 mm Horizontal: $180 \mathrm{~mm}$ ", was assigned. For the material of the curtain walls, BIM components published by Schueco, hosted in the BIM object library, "Façade UCC 65 SG" for the walls and "Sliding System ASS 77 PD" for the opening were edited to fit plan specifications and assigned. External wall types 2, 3, and the internal walls were recreated in the BIM model according to the plan specifications. This building is designed with a steel framing structure; therefore, a steel material was assigned to each framing component in the BIM model. The square hollow section and universal columns in the model were assigned the "Metal-Steel 50-355" from the Revit material library. The circular hollow sections, multibeam purlins and rails, parallel flange channels (PFC) plan, rectangular and square hollow sections and universal beams were assigned the "Metal-Steel-S275" material. The "Cast-in-Place Concrete" material was assigned to the floor, foundation slab, wall foundation and pile cap components. All of these materials are depicted in Table 2. 
Table 2. Material list of structural material used in Lidl standard design supermarkets in the UK with the added material in Revit $^{\circledR}$.

\begin{tabular}{|c|c|c|c|}
\hline Component & Assigned Component & Assigned Material in BIM Model & Material Layer Details \\
\hline Floor & $\begin{array}{l}\text { Floor: Floor-Upper } 160 \\
\text { mmConc-CorusComFlor } 51\end{array}$ & Concrete $15 \mathrm{MPa}$ & \\
\hline Foundation Slab & $\begin{array}{l}\text { Foundation Slab: Standard } \\
\text { with tiles }\end{array}$ & Cast-in-Place Concrete & \\
\hline Pile Cap & Pile Cap: $1500 \times 1500 \times 500$ & Cast-in-Place Concrete & \\
\hline Wall Foundation & Wall Foundation: Standard & Cast-in-Place Concrete & \\
\hline \multirow{3}{*}{ Basic Roof } & \multirow{3}{*}{$\begin{array}{c}\text { Kingspan Insulated } \\
\text { Panels-Metal Insulating } \\
\text { Sandwich Panel Roofing } \\
\text { System-Quad Core } \\
\text { Trapezoidal Roof } \\
\text { Panel-KS1000RW } 120 \mathrm{~mm}\end{array}$} & $\begin{array}{l}\text { NBS Kingspan Insulated Panels PIR } \\
\text { Foam Board PIR Insulation }\end{array}$ & \\
\hline & & $\begin{array}{c}\text { NBS Kingspan Insulated Panels Steel } \\
\text { Profiled Sheet Quad-Core Trapezoidal } \\
\text { Roof Panel KS1000RW External Weather } \\
\text { Sheet }\end{array}$ & \\
\hline & & $\begin{array}{c}\text { NBS Kingspan Insulated Panels Steel } \\
\text { Profiled Sheet Quad-Core Trapezoidal } \\
\text { Roof Panel KS1000RW Internal Liner } \\
\text { Sheet }\end{array}$ & \\
\hline \multirow{2}{*}{ Basic Roof } & \multirow{2}{*}{$\begin{array}{l}\text { Metal single skin, } 125 \mathrm{~mm} \\
\text { insulation }\end{array}$} & Default Roof-metal single skin & \\
\hline & & Default Roof-Generic insulation $125 \mathrm{~mm}$ & \\
\hline $\begin{array}{l}\text { Square Hollow } \\
\text { Sections-Column }\end{array}$ & $\begin{array}{c}\text { RHS200 } \times 100 \times 6.3 \\
\text { SHS } 80 \times 80 \times 8 \\
\text { SHS150 } \times 150 \times 5 \\
\text { SHS200 } \times 200 \times 6.3 \\
\text { SHS250 } \times 250 \times 6.3\end{array}$ & Metal-Steel 50-355 & \\
\hline $\begin{array}{l}\text { UC-Universal } \\
\text { Columns-Column }\end{array}$ & $\begin{array}{l}\mathrm{UC} 152 \times 152 \times 23 \\
\mathrm{UC} 152 \times 152 \times 30 \\
\mathrm{UC} 203 \times 203 \times 46\end{array}$ & Metal-Steel 50-355 & \\
\hline Circular Hollow Sections & CHS114.3 × 5 & Metal-Steel-S275 & \\
\hline $\begin{array}{l}\text { Light Gauge-Kingspan } \\
\text { Multibeam Purlins }\end{array}$ & M205065180 & Metal-Steel-S275 & \\
\hline $\begin{array}{l}\text { Light Gauge-Kingspan } \\
\text { Multibeam Rails }\end{array}$ & M205065180 & Metal-Steel-S275 & \\
\hline $\begin{array}{l}\text { PFC-Parallel Flange } \\
\text { Channels }\end{array}$ & $\begin{array}{l}\text { PFC150 } \times 75 \times 18 \\
\text { PFC200 } \times 75 \times 23\end{array}$ & Metal-Steel-S275 & \\
\hline Plate & Plate: $10 \times 100$ PLT & Metal-Steel-S275 & \\
\hline $\begin{array}{l}\text { Rectangular Hollow } \\
\text { Sections }\end{array}$ & $\begin{array}{l}\text { RHS140 × } 80 \times 6.3 \\
\text { RHS200 } \times 100 \times 6.3\end{array}$ & Metal-Steel-S275 & \\
\hline Square Hollow Sections & $\begin{array}{c}\text { SHS150 } \times 150 \times 5 \\
\text { SHS180 × } 180 \times 6.3\end{array}$ & Metal-Steel-S275 & \\
\hline UB-Universal Beams & $\begin{array}{l}\text { UB203 } \times 133 \times 25 \\
\text { UB203 } \times 133 \times 25 \\
\text { UB254 } \times 146 \times 31 \\
\text { UB305 } \times 165 \times 54 \\
\text { UB406 } \times 140 \times 39 \\
\text { UB533 } \times 210 \times 122 \\
\text { UB610 } \times 229 \times 101 \\
\text { UB686 } \times 254 \times 125\end{array}$ & Metal-Steel-S275 & \\
\hline
\end{tabular}


Table 2. Cont.

\begin{tabular}{|c|c|c|c|}
\hline Component & Assigned Component & Assigned Material in BIM Model & Material Layer Details \\
\hline \multirow{3}{*}{ External wall type 1} & \multirow{3}{*}{$\begin{array}{c}\text { Flamstyl S } \\
\text { ArcelorMittal-1000 mm } \\
\text { Horizontal: } 180 \mathrm{~mm}\end{array}$} & ArcelorMittal-Mineral Wool & Thermoplastic Resin \\
\hline & & $\begin{array}{l}\text { Pre-painted Steel ArcelorMittal } \\
\text { Construction HAIRULTRA }\end{array}$ & $\begin{array}{l}\text { Steel, paint finish, ivory, } \\
\text { glossy }\end{array}$ \\
\hline & & $\begin{array}{l}\text { Pre-painted Steel ArcelorMittal } \\
\text { Construction INTÉRIEUR }\end{array}$ & $\begin{array}{l}\text { Polymethyl } \\
\text { Methacrylate }\end{array}$ \\
\hline \multirow{3}{*}{ External wall type 2} & \multirow{3}{*}{ External wall type 2} & Portland Cement Concrete & Concrete \\
\hline & & Insulation/Support Frame & \\
\hline & & Paint-White Lining & \\
\hline \multirow{2}{*}{ External wall type 3} & \multirow{2}{*}{ External wall type 3} & Cladding, Vertical Ribbed & \\
\hline & & Insulation/Support Frame & \\
\hline \multirow{3}{*}{ Internal wall type 1} & \multirow{3}{*}{$\begin{array}{l}\text { Internal wall type } 1 \mathrm{~A} \\
\quad(300 \mathrm{~mm}) \\
\text { Internal wall type } 1 \mathrm{~B} \\
\quad(399 \mathrm{~mm}) \\
\text { Internal wall type } 1 \mathrm{C} \\
\quad(428 \mathrm{~mm})\end{array}$} & Gypsum Wall Board & \\
\hline & & $\begin{array}{l}\text { Light gauge steel framing, thermal air } \\
\text { layer }\end{array}$ & Metal Stud Layer \\
\hline & & Rock Wool & \\
\hline \multirow{3}{*}{ Internal wall type 2} & \multirow{3}{*}{ Internal wall type 2} & Gypsum Wall Board & \\
\hline & & $\begin{array}{l}\text { Light gauge steel framing, thermal air } \\
\text { layer }\end{array}$ & Metal Stud Layer \\
\hline & & Rock Wool & \\
\hline \multirow{3}{*}{ Internal wall type 3} & \multirow{3}{*}{ Internal wall type 3} & Gypsum Wall Board & \\
\hline & & $\begin{array}{c}\text { Light gauge steel framing, thermal air } \\
\text { layer }\end{array}$ & Metal Stud Layer \\
\hline & & Rock Wool & \\
\hline \multirow{3}{*}{ Internal wall type 4} & \multirow{3}{*}{ Internal wall type 4} & Gypsum Wall Board & \\
\hline & & $\begin{array}{c}\text { Light gauge steel framing, thermal air } \\
\text { layer }\end{array}$ & Metal Stud Layer \\
\hline & & Rock Wool & \\
\hline \multirow{3}{*}{ Internal wall type 5} & \multirow{3}{*}{ Internal wall type 5} & Gypsum Wall Board & \\
\hline & & $\begin{array}{c}\text { Light gauge steel framing, thermal air } \\
\text { layer }\end{array}$ & Metal Stud Layer \\
\hline & & Rock Wool & \\
\hline \multirow{2}{*}{ Internal wall type 6} & \multirow{2}{*}{ Internal wall type 6} & Common brick & \\
\hline & & Plaster & \\
\hline \multirow{2}{*}{ Internal wall type 7} & \multirow{2}{*}{ Internal wall type 7} & Concrete-Precast Concrete- $35 \mathrm{MPa}$ & \\
\hline & & Plaster & \\
\hline \multirow{2}{*}{ Curtain wall opening } & \multirow{2}{*}{$\begin{array}{c}\text { Schueco ASS } 77 \text { PD-HI } \\
\text { Family-2D-1 OF-150 mm: } \\
\text { Type 2D }\end{array}$} & Soda Lime Glass & \\
\hline & & Aluminium & \\
\hline \multirow{2}{*}{ Curtain wall type 1} & \multirow{2}{*}{$\begin{array}{l}\text { Schueco Façade UCC } 65 \text { SG } \\
\text { Family-03: Type } 1\end{array}$} & Soda Lime Glass & \\
\hline & & Aluminium & \\
\hline \multirow{2}{*}{ Curtain wall type 2} & \multirow{2}{*}{$\begin{array}{l}\text { Schueco Façade UCC } 65 \text { SG } \\
\text { Family-13: Type } 1\end{array}$} & Soda Lime Glass & \\
\hline & & Aluminium & \\
\hline
\end{tabular}




\section{BIM Delimitations}

Based on the plan specifications, $91 \%$ of the internal walls in the model were assigned stud wall layers of various thicknesses. The material was initially designed using the internal Revit material "Metal Stud Layer: Light gauge steel framing, thermal air layer". However, this Revit material does not account for the gaps between each individual steel frame. The material and subsequent unit weight attributed to this layer is steel leading to a discrepancy in the material weight for these walls. The internal walls were modelled individually, as depicted in Table 3, in the software to mitigate this discrepancy and take-off the correct material weight (Figure 5). When taking this approach, the total weight of the stud layer was reduced from $1,466,265.9 \mathrm{~kg}$ to $169,630.2 \mathrm{~kg}$, signifying an $88.4 \%$ reduction. Had the LCA been carried out using the former values, an additional 1,296,635.60 kg of steel would be calculated (Table 4), thus, demonstrating the necessity of this step to ensure an accurate result.

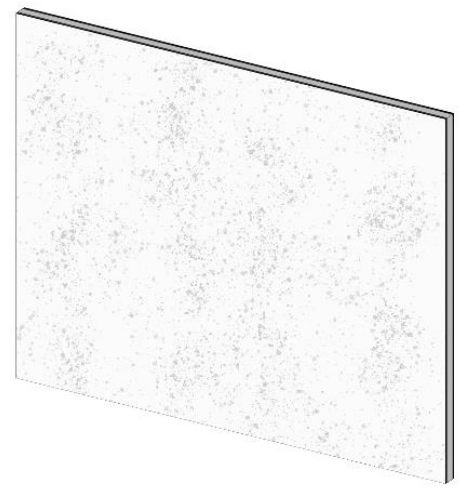

Initial Component

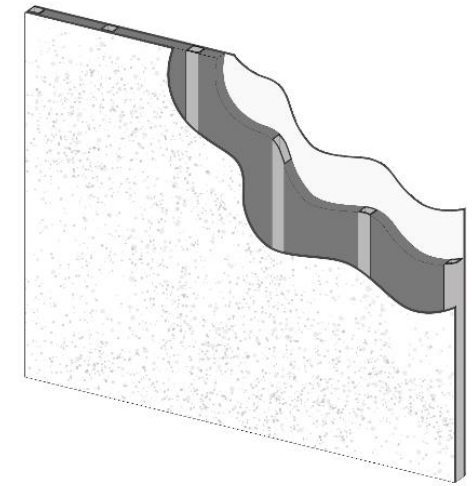

Modelled Component
| Gypsum wall board

Metal Stud Layer

Rock wool insulaiton

Figure 5. Schematic image of internal wall with internal stud layer modelled in Revit ${ }^{\circledR}$.

Table 3. Internal wall, layer details in Revit ${ }^{\circledR}$ BIM software.

\begin{tabular}{|c|c|c|c|c|c|}
\hline Wall Type & \multicolumn{5}{|c|}{ Layer Details of Modelled Material in Revit ${ }^{\circledR}$} \\
\hline \multirow{10}{*}{$\begin{array}{c}\text { (A) Internal wall type } 1 \mathrm{~A} \text {, layer details in } \\
\text { Revit }^{\circledR} \text { BIM software }\end{array}$} & & Function & Material & Thickness & Wraps \\
\hline & 1 & Finish 2 [5] & Gypsum Wall & 0.0150 & $\nabla$ \\
\hline & 2 & Core Boundar & Layers Above & 0.0000 & \\
\hline & 3 & Structure [1] & Rock Wool & 0.0818 & $\square$ \\
\hline & 4 & Structure [1] & Metal Stud La & 0.0102 & $\square$ \\
\hline & 5 & Thermal/Air Lay & < By Category & 0.0860 & $\square$ \\
\hline & 6 & Structure [1] & Metal Stud La & 0.0102 & $\square$ \\
\hline & 7 & Structure [1] & Rock Wool & 0.0818 & 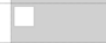 \\
\hline & 8 & Core Boundar & Layers Below & 0.0000 & \\
\hline & 9 & Finish $2[5]$ & Gypsum Wall & 0.0150 & $\square$ \\
\hline \multirow{10}{*}{$\begin{array}{c}\text { (B) Internal wall type 1B, layer details in } \\
\text { Revit }^{\circledR} \text { BIM software }\end{array}$} & & Function & Material & Thickness & Wraps \\
\hline & 1 & Finish 2 [5] & Gypsum Wall & 0.0150 & $\square$ \\
\hline & 2 & Core Boundar & Layers Above & 0.0000 & \\
\hline & 3 & Structure [1] & Rock Wool & 0.0818 & $\square$ \\
\hline & 4 & Structure [1] & Metal Stud La & 0.0102 & $\square$ \\
\hline & 5 & Thermal/Air Lay & $<$ By Category & 0.1850 & $\square$ \\
\hline & 6 & Structure [1] & Metal Stud La & 0.0102 & $\square$ \\
\hline & 7 & Structure [1] & Rock Wool & 0.0818 & $\square$ \\
\hline & 8 & Core Boundar & Layers Below & 0.0000 & \\
\hline & 9 & Finish 2 [5] & Gypsum Wall & 0.0150 & $\square$ \\
\hline
\end{tabular}


Table 3. Cont.

\begin{tabular}{|c|c|c|c|c|c|}
\hline Wall Type & \multicolumn{5}{|c|}{ Layer Details of Modelled Material in Revit ${ }^{\circledR}$} \\
\hline \multirow{10}{*}{$\begin{array}{c}\text { (C) Internal wall type } 1 C \text {, layer details in } \\
\text { Revit }^{\circledR} \text { BIM software }\end{array}$} & & Function & Material & Thickness & Wraps \\
\hline & 1 & Finish 2 [5] & Gypsum Wall & 0.0150 & $\square$ \\
\hline & 2 & Core Boundar & Layers Above & 0.0000 & \\
\hline & 3 & Structure [1] & Rock Wool & 0.0818 & 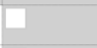 \\
\hline & 4 & Structure [1] & Metal Stud La & 0.0102 & 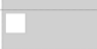 \\
\hline & 5 & Thermal/Air Lay & $<$ By Category & 0.2140 & $\square$ \\
\hline & 6 & Structure [1] & Metal Stud La & 0.0102 & $\square$ \\
\hline & 7 & Structure [1] & Rock Wool & 0.0818 & $\square$ \\
\hline & 8 & Core Boundar & Layers Below & 0.0000 & \\
\hline & 9 & Finish $2[5]$ & Gypsum Wall & 0.0150 & $\square$ \\
\hline
\end{tabular}

(D) Internal wall type 2, layer details in Revit ${ }^{\circledR}$ BIM software

(E) Internal wall type 3, layer details in Revit ${ }^{\circledR}$ BIM software

(F) Internal wall type 4, layer details in Revit ${ }^{\circledR}$ BIM software

\begin{tabular}{|l|l|l|l|l|}
\hline & \multicolumn{1}{|c|}{ Function } & \multicolumn{1}{|c|}{ Material } & \multicolumn{1}{|c|}{ Thickness } & Wraps \\
\hline 1 & Finish 1 [4] & Gypsum Wall & 0.0150 & $\square$ \\
\hline 2 & Core Boundar & Layers Above & $\mathbf{0 . 0 0 0 0}$ & \\
\hline 3 & Structure [1] & Rock Wool & 0.1200 & \\
\hline 4 & Structure [1] & Metal Stud La & 0.0260 & \\
\hline 5 & Core Boundar & Layers Below & $\mathbf{0 . 0 0 0 0}$ & \\
\hline 6 & Finish 1 [4] & Gypsum Wall & 0.0150 & $\square$ \\
\hline
\end{tabular}

\begin{tabular}{|l|l|l|l|l}
\hline & \multicolumn{1}{|c|}{ Function } & \multicolumn{1}{|c|}{ Material } & \multicolumn{1}{|c|}{ Thickness } & Wraps \\
\hline 1 & Finish 1 [4] & Gypsum Wall B & 0.0150 & $\square$ \\
\hline 2 & Core Boundary & Layers Above W $\mathbf{0 . 0 0 0 0}$ & \\
\hline 3 & Structure [1] & Rock Wool & 0.0641 & \\
\hline 4 & Structure [1] & Metal Stud Lay & 0.0059 & \\
\hline 5 & Core Boundary & Layers Below W $\mathbf{0 . 0 0 0 0}$ & \\
\hline 6 & Finish 1 [4] & Gypsum Wall B & 0.0150 & $\square$ \\
\hline
\end{tabular}

\begin{tabular}{|l|l|l|l|l}
\hline & \multicolumn{1}{|c|}{ Function } & \multicolumn{1}{|c|}{ Material } & \multicolumn{1}{|c|}{ Thickness } & Wraps \\
\hline 1 & Finish 1 [4] & Gypsum Wall & 0.0150 & $\square$ \\
\hline 2 & Core Boundar & Layers Above & $\mathbf{0 . 0 0 0 0}$ & \\
\hline 3 & Structure [1] & Rock Wool & 0.0641 & \\
\hline 4 & Structure [1] & Metal Stud La & 0.0059 & \\
\hline 5 & Core Boundar & Layers Below & $\mathbf{0 . 0 0 0 0}$ & \\
\hline
\end{tabular}

\begin{tabular}{|l|l|l|l|l}
\hline & \multicolumn{1}{|c|}{ Function } & \multicolumn{1}{|c|}{ Material } & \multicolumn{1}{c|}{ Thickness } & Wraps \\
\hline 1 & Finish 1 [4] & Gypsum Wall & 0.0150 & $\square$ \\
\hline 2 & Core Boundar & Layers Above & $\mathbf{0 . 0 0 0 0}$ & \\
\hline 3 & Structure [1] & Metal Stud La & 0.0102 & \\
\hline 4 & Structure [1] & Rock Wool & 0.0818 & \\
\hline $\mathbf{5}$ & Core Boundar & Layers Below & $\mathbf{0 . 0 0 0 0}$ & \\
\hline
\end{tabular}

(G) Internal wall type 5, layer details in Revit ${ }^{\circledR}$ BIM software 
Table 4. Comparison of stud wall weight when using Revit ${ }^{\circledR}$ material vs. modelled component.

\begin{tabular}{|c|c|c|c|c|}
\hline Wall Type & $\begin{array}{c}\text { Assigned Material in BIM } \\
\text { Model }\end{array}$ & Unit Weight kg/m ${ }^{3}$ & $\begin{array}{l}\text { Initial Component } \\
\text { Weight (kg) }\end{array}$ & $\begin{array}{l}\text { Modelled Component } \\
\text { Weight (kg) }\end{array}$ \\
\hline Internal wall type 1A & $\begin{array}{c}\text { Light gauge steel framing, } \\
\text { thermal air layer }\end{array}$ & $7850.0 \mathrm{~kg} / \mathrm{m}^{3}$ & $583,049.2 \mathrm{~kg}$ & $64,644.6 \mathrm{~kg}$ \\
\hline Internal wall type $1 \mathrm{~B}$ & $\begin{array}{l}\text { Light gauge steel framing, } \\
\text { thermal air layer }\end{array}$ & $7850.0 \mathrm{~kg} / \mathrm{m}^{3}$ & $6758.8 \mathrm{~kg}$ & $753.6 \mathrm{~kg}$ \\
\hline Internal wall type $1 \mathrm{C}$ & $\begin{array}{l}\text { Light gauge steel framing, } \\
\text { thermal air layer }\end{array}$ & $7850.0 \mathrm{~kg} / \mathrm{m}^{3}$ & $420,240.7 \mathrm{~kg}$ & $46,589.6 \mathrm{~kg}$ \\
\hline Internal wall type 2 & $\begin{array}{c}\text { Light gauge steel framing, } \\
\text { thermal air layer }\end{array}$ & $7850.0 \mathrm{~kg} / \mathrm{m}^{3}$ & $141,260.3 \mathrm{~kg}$ & $25,143.5 \mathrm{~kg}$ \\
\hline Internal wall type 3 & $\begin{array}{l}\text { Light gauge steel framing, } \\
\text { thermal air layer }\end{array}$ & $7850.0 \mathrm{~kg} / \mathrm{m}^{3}$ & $38,849.5 \mathrm{~kg}$ & $3289.1 \mathrm{~kg}$ \\
\hline Internal wall type 4 & $\begin{array}{c}\text { Light gauge steel framing, } \\
\text { thermal air layer }\end{array}$ & $7850.0 \mathrm{~kg} / \mathrm{m}^{3}$ & $52,948.1 \mathrm{~kg}$ & $4458.8 \mathrm{~kg}$ \\
\hline Internal wall type 5 & $\begin{array}{c}\text { Light gauge steel framing, } \\
\text { thermal air layer }\end{array}$ & $7850.0 \mathrm{~kg} / \mathrm{m}^{3}$ & $223,159.2 \mathrm{~kg}$ & $24,751.0 \mathrm{~kg}$ \\
\hline
\end{tabular}

\subsection{Material Weight}

The material weight for each building element was derived from a quantity take-off (QTO) of the BIM model. The categories included in this take-off were Floors, Curtain panels, Roofs, Structural Columns, Structural Foundation, Structural Framing, Walls, and Windows. A material take-off process provides a complete and accurate weight for each composing materials for the elements within these categories (Table 5). The weight for the mechanical ducts cannot be derived by this method. However, the total size of the mechanical elements can be obtained from a mechanical schedule take-off in Revit ${ }^{\circledR}$. The weight for each component can then be calculated using Equations (2) and (3).

Rectangular duct mass $=2 \times($ Height + Width $) \times$ length $\times$ thickness $\times$ materials density

$$
\text { Round duct mass }=\text { length } \times \pi \times \text { diameter } \times \text { thickness } \times \text { material density }
$$

In these formulas, the density of the materials is $7800 \mathrm{~kg} / \mathrm{m}^{3}$. Additionally, the minimum thickness is assigned to the ducts. The minimum thickness for each component is determined by size according to DW/144 specifications [21]. This process is repeated for the mechanical duct fittings using the values obtained from a duct fitting schedule take-off in Revit ${ }^{\circledR}$.

Table 5. Material weight of building components in Lidl supermarket standard design.

\begin{tabular}{clcc}
\hline Category & \multicolumn{1}{c}{ Family and Type } & Material: Name & Material: Weight (kg) \\
\hline \multirow{2}{*}{ Floors } & $\begin{array}{l}\text { Floor: Floor-Upper_160 } \\
\text { mmConc-CorusComFlor51 }\end{array}$ & Concrete, Cast-in-Place-C15 & $14,674.397 \mathrm{~kg}$ \\
\hline $\begin{array}{c}\text { Structural } \\
\text { Foundations }\end{array}$ & Foundation Slab: Standard with tiles & Default Mass Floor & $1190.908 \mathrm{~kg}$ \\
\cline { 2 - 4 } & Pile Cap: $1500 \times 1500 \times 500$ & $\begin{array}{c}\text { Concrete-Cast-in-Place } \\
\text { Concrete }\end{array}$ & $97,024.061 \mathrm{~kg}$ \\
\cline { 2 - 4 } & Wall Foundation: Standard & Concrete, Cast In Situ & $4.813 \mathrm{~kg}$ \\
\hline \multirow{2}{*}{ Mechanical } & $\begin{array}{l}\text { Rectangular Duct: Standard, Fitting } \\
\text { Round Duct: Standard, Fitting }\end{array}$ & Steel & $4528.741 \mathrm{~kg}$ \\
\hline
\end{tabular}


Table 5. Cont.

\begin{tabular}{|c|c|c|c|c|}
\hline Category & \multicolumn{2}{|c|}{ Family and Type } & Material: Name & Material: Weight (kg) \\
\hline \multirow{5}{*}{ Roofs } & \multirow{3}{*}{\multicolumn{2}{|c|}{$\begin{array}{l}\text { Kingspan Insulated Panels-Metal Insulating } \\
\text { Sandwich Panel Roofing System-Quad Core } \\
\text { Trapezoidal Roof Panel-KS1000RW } 120 \text { mm }\end{array}$}} & $\begin{array}{l}\text { NBS_KingspanInsulatedPanels_ } \\
\text { PIRFoamBoard_PIRInsulation }\end{array}$ & $12,965.762 \mathrm{~kg}$ \\
\hline & & & $\begin{array}{l}\text { NBS_KingspanInsulatedPanels_ } \\
\text { SteelProfiledSheet_QuadCore } \\
\text { TrapezoidalRoofPanel_KS1000RW__ } \\
\quad \text { ExternalWeatherSheet }\end{array}$ & $19,720.414 \mathrm{~kg}$ \\
\hline & & & $\begin{array}{l}\text { NBS_KingspanInsulatedPanels_ } \\
\text { SteelProfiledSheet_QuadCore } \\
\text { TrapezoidalRoofPanel_KS1000RW_ } \\
\quad \text { InternalLinerSheet }\end{array}$ & $29,468.400 \mathrm{~kg}$ \\
\hline & \multirow{2}{*}{\multicolumn{2}{|c|}{ Metal single skin, $125 \mathrm{~mm}$ insulation }} & $\begin{array}{l}\text { Default Roof-metal single } \\
\text { skin }\end{array}$ & $43.790 \mathrm{~kg}$ \\
\hline & & & $\begin{array}{l}\text { Default Roof-Generic } \\
\text { insulation } 125 \mathrm{~mm}\end{array}$ & $178.040 \mathrm{~kg}$ \\
\hline Structural Columns & $\begin{array}{l}\text { RHS200 } \times 100 \times 6.3 \\
\text { SHS80 × } 80 \times 8 \\
\text { SHS150 × } 150 \times 5 \\
\text { SHS200 × } 200 \times 6.3 \\
\text { SHS250 } \times 250 \times 6.3\end{array}$ & $\begin{array}{l}\mathrm{UB} 203 \times 133 \times 25 \\
\mathrm{UC} 152 \times 152 \times 23 \\
\mathrm{UC} 152 \times 152 \times 30 \\
\mathrm{UC} 203 \times 203 \times 46\end{array}$ & Metal-Steel 50-355 & $20,982.768 \mathrm{~kg}$ \\
\hline Structural Framing & $\begin{array}{l}\text { CHS114.3 ×5 } \\
\text { M205065180 } \\
\text { M205065180 } \\
\text { PFC150 × } \times 15 \\
\text { PFC200 } \times 75 \times 23 \\
10 \times 100 \text { PLT } \\
\text { RHS140 × } 80 \times 6.3 \\
\text { RHS200 × } 100 \times 6.3 \\
\text { SHS150 } \times 150 \times 5 \\
\text { SHS180 } \times 180 \times 6.3\end{array}$ & $\begin{array}{l}\mathrm{UB} 203 \times 133 \times 25 \\
\mathrm{UB} 203 \times 133 \times 25 \\
\mathrm{UB} 254 \times 146 \times 31 \\
\mathrm{UB} 305 \times 165 \times 54 \\
\mathrm{UB} 406 \times 140 \times 39 \\
\mathrm{UB} 333 \times 210 \times 122 \\
\mathrm{UB} 610 \times 229 \times 101 \\
\mathrm{UB} 686 \times 254 \times 125 \\
\mathrm{UC} 152 \times 152 \times 23\end{array}$ & Metal-Steel-S275 & $64,730.549 \mathrm{~kg}$ \\
\hline \multirow{10}{*}{ Walls } & \multirow{4}{*}{\multicolumn{2}{|c|}{ External wall type 1}} & ArcelorMittal-Mineral Wool & $244,027.357 \mathrm{~kg}$ \\
\hline & & & $\begin{array}{c}\text { Prepainted_Steel- } \\
\text { ArcelorMittal_Construction_- } \\
\text { HAIRULTRA-35 }\end{array}$ & $27,644.390 \mathrm{~kg}$ \\
\hline & & & $\begin{array}{c}\text { Prepainted_Steel- } \\
\text { ArcelorMittal_Construction_- } \\
\text { INTÉRIEUR }\end{array}$ & $3213.457 \mathrm{~kg}$ \\
\hline & & & Cladding, Vertical Ribbed & $11,376.568 \mathrm{~kg}$ \\
\hline & \multirow{3}{*}{\multicolumn{2}{|c|}{ External wall type 2}} & Concrete & $9764.194 \mathrm{~kg}$ \\
\hline & & & Insulation/Support Frame & $8.912 \mathrm{~kg}$ \\
\hline & & & Paint-White Lining & $0.557 \mathrm{~kg}$ \\
\hline & \multirow{2}{*}{\multicolumn{2}{|c|}{ External wall type 3}} & Cladding, Vertical Ribbed & $6747.893 \mathrm{~kg}$ \\
\hline & & & Insulation/Support Frame & $19.551 \mathrm{~kg}$ \\
\hline & \multicolumn{2}{|c|}{$\begin{array}{l}\text { Internal wall type } 1 \mathrm{~A}(300 \mathrm{~mm}) \\
\text { Internal wall type } 1 \mathrm{~B}(399 \mathrm{~mm}) \\
\text { Internal wall type } 1 \mathrm{C}(428 \mathrm{~mm})\end{array}$} & Default Wall & $768.253 \mathrm{~kg}$ \\
\hline
\end{tabular}


Table 5. Cont.

\begin{tabular}{|c|c|c|c|c|}
\hline Category & \multicolumn{2}{|c|}{ Family and Type } & Material: Name & $\begin{array}{c}\text { Material: Weight } \\
\text { (kg) }\end{array}$ \\
\hline & $\begin{array}{l}\text { Internal wall type } 1 \mathrm{~A} \\
(300 \mathrm{~mm}) \\
\text { Internal wall type } 1 \mathrm{~B} \\
(399 \mathrm{~mm}) \\
\text { Internal wall type } 1 \mathrm{C} \\
(428 \mathrm{~mm}) \\
\text { Internal wall type } 2\end{array}$ & $\begin{array}{l}\text { Internal wall type } 3 \\
\text { Internal wall type } 4 \\
\text { Internal wall type } 5\end{array}$ & Gypsum Wall Board & $36,163.635 \mathrm{~kg}$ \\
\hline & $\begin{array}{l}\text { Internal wall type } 1 \mathrm{~A} \\
(300 \mathrm{~mm}) \\
\text { Internal wall type } 1 \mathrm{~B} \\
(399 \mathrm{~mm}) \\
\text { Internal wall type } 1 \mathrm{C} \\
(428 \mathrm{~mm}) \\
\text { Internal wall type } 2\end{array}$ & $\begin{array}{l}\text { Internal wall type } 3 \\
\text { Internal wall type } 4 \\
\text { Internal wall type } 5\end{array}$ & Metal Stud Layer & $169,630.170 \mathrm{~kg}$ \\
\hline & $\begin{array}{l}\text { Internal wall type } 1 \mathrm{~A} \\
(300 \mathrm{~mm}) \\
\text { Internal wall type } 1 \mathrm{~B} \\
(399 \mathrm{~mm}) \\
\text { Internal wall type } 1 \mathrm{C} \\
(428 \mathrm{~mm}) \\
\text { Internal wall type } 2\end{array}$ & $\begin{array}{l}\text { Internal wall type } 3 \\
\text { Internal wall type } 4 \\
\text { Internal wall type } 5\end{array}$ & Rock Wool & $33,027.235 \mathrm{~kg}$ \\
\hline & Internal wall type 6 & & Brick, Common & $25,933.089 \mathrm{~kg}$ \\
\hline & $\begin{array}{l}\text { Internal wall type } 6 \\
\text { Internal wall type } 7\end{array}$ & & Plaster & $4467.497 \mathrm{~kg}$ \\
\hline & Internal wall type 7 & & $\begin{array}{l}\text { Concrete-Precast Concrete-35 } \\
\mathrm{MPa}\end{array}$ & $12,790.706 \mathrm{~kg}$ \\
\hline \multirow{2}{*}{ Curtain wall } & \multicolumn{2}{|c|}{$\begin{array}{l}\text { Schueco ASS } 77 \text { PD-HI Family-2D-1 OF-150 } \\
\text { mm: Type 2D } \\
\text { Schueco Façade UCC } 65 \text { SG Family-03: Type } 1 \\
\text { Schueco Façade UCC } 65 \text { SG Family-13: Type } 1\end{array}$} & Alu & $2678.138 \mathrm{~kg}$ \\
\hline & \multicolumn{2}{|c|}{$\begin{array}{l}\text { Schueco ASS } 77 \text { PD-HI Family-2D-1 OF-150 } \\
\text { mm: Type 2D } \\
\text { Schueco Façade UCC } 65 \text { SG Family-03: Type } 1 \\
\text { Schueco Façade UCC } 65 \text { SG Family-13: Type } 1\end{array}$} & Glass & $8094.866 \mathrm{~kg}$ \\
\hline Windows & \multicolumn{2}{|c|}{ AWS60BD 2 Vents: AWS60BD 2 Vents } & Glass & $191.179 \mathrm{~kg}$ \\
\hline & \multicolumn{2}{|c|}{ Total } & & $862,078.845 \mathrm{~kg}$ \\
\hline
\end{tabular}

\subsection{Embodied Carbon Calculations}

In this study, the method of assigning embodied carbon factors was defined by choice of ECF database or source and the choice of appropriate data within those databases or sources.

The carbon factors in this study were derived from two primary databases. The first database used was the GHG conversion factors dataset published by the UK Department of Energy \& Climate Change [9]. In this dataset, the ECFs are broken down into 40 different categories, with construction materials broken down into the following fourteen categories: Aggregates, Average construction, Asbestos, Asphalt, Bricks, Concrete, Insulation, Metals, Soils, Mineral oil, Plasterboard, Tyres, and Wood. The second database used was the Embodied Carbon-ICE Database published by the Institute of Civil Engineering [19]. Furthermore, ECFs were also sourced from published data and data derived from literature.

In this effect, two approaches were taken in the assignment of appropriate embodied carbon factors. The first approach was to assign ECFs to materials based on the general 
category of the material. The second approach was to assign ECFs to each material to be the closest to materials details.

The first set of calculations were carried out using the UK Department of Energy \& Climate Change database. Given the limited nature of this database, the first approach in assigning ECFs was taken. The second set of calculations also used the first approach of ECF assignment using the ICE database, which provides more vast data. The detailed or second approach was taken for the third set of calculations. In this approach, the ECF assigned to each material was chosen to be the closest to materials details. In this approach, the majority of the ECFs were taken from the ICE database. However, for some materials, published data and data derived from literature was used for a more accurate ECF value (Figure 6).

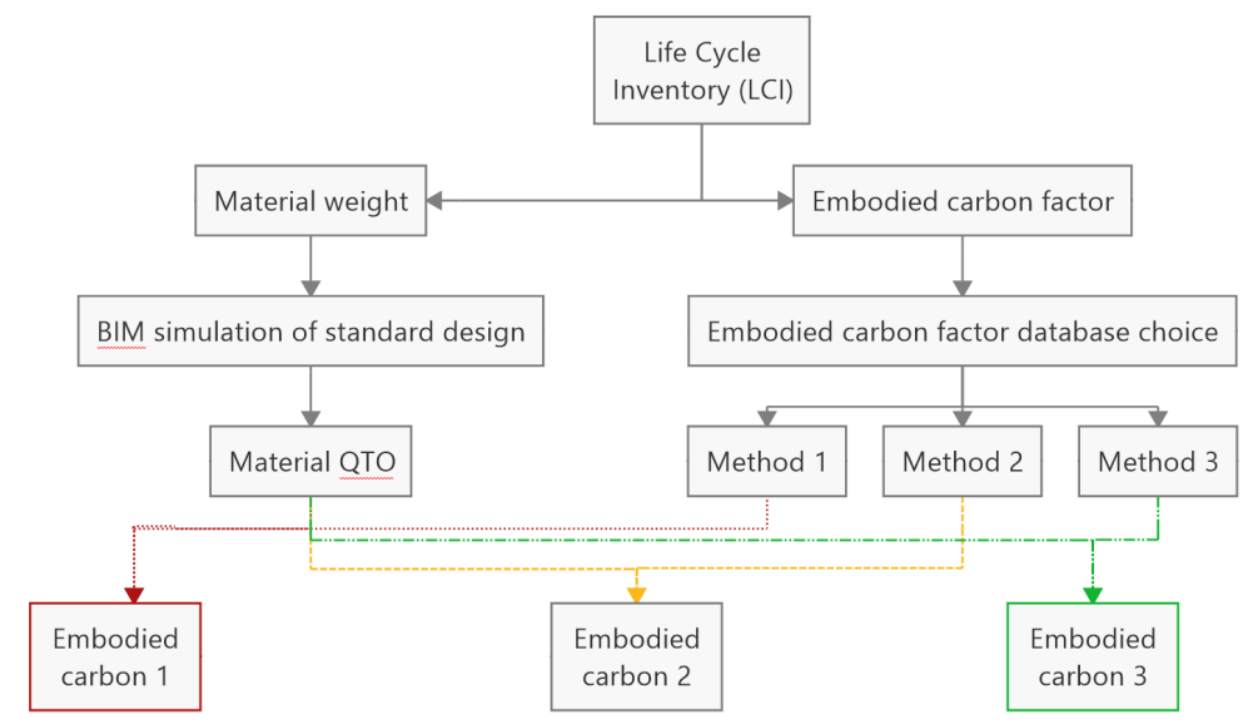

Figure 6. Flowchart depicting the methodology.

In method one, the fourteen ECF values in the construction category of the database were assigned. The "Concrete" ECF was assigned to all concrete materials of the building components, including materials in the floor, structural foundation, and internal wall elements. The ECF value attributed to concrete in this database is $0.135 \mathrm{kgCO} 2 \mathrm{e} / \mathrm{kg}$. In methods 2 and 3, "ICE-Concrete, General" was assigned to the materials in the structural foundation and floor elements. In this database the ECF value for this material is $0.103 \mathrm{kgCO} 2 \mathrm{e} / \mathrm{kg}$, while ECF assigned to the concrete material for internal wall element in method two was "ICE-precast ACC block" and in method three "ICE-Concrete 35/45", their values respectively $0.28 \mathrm{kgCO} 2 \mathrm{e} / \mathrm{kg}$ and $0.1609 \mathrm{kgCO} 2 \mathrm{e} / \mathrm{kg}$.

The "Metals" ECF was assigned to all metal materials assigned to the building components, including steel, aluminium, cladding, and sheet metals in the roof, walls, curtain wall, windows, mechanical ducts, and structural framing elements. The carbon factor for all metals in this database is $4.769 \mathrm{kgCO} 2 \mathrm{e} / \mathrm{kg}$. In methods two and three, "ICE-Steel", with a value of $2.970 \mathrm{kgCO} 2 \mathrm{e} / \mathrm{kg}$, was assigned to the internal wall metal materials. This value was also assigned to the roof and external wall metal materials in method two. While "ICE-Steel, organic coated sheet" with a value of $3.060 \mathrm{kgCO} 2 \mathrm{e} / \mathrm{kg}$ was assigned to these elements in method three. "ICE-Steel pipe" with a value of $3.02 \mathrm{CO} 2 \mathrm{e} / \mathrm{kg}$ was assigned to the mechanical duct material. "ICE-aluminium sheet general EU including imports" with a value of $3.291 \mathrm{kgCO} 2 \mathrm{e} / \mathrm{kg}$ were assigned to the external wall and roof metal materials in methods two and three. "ICE-Steel section" with a value of $1.55 \mathrm{kgCO} 2 \mathrm{e} / \mathrm{kg}$ was assigned to the structural framing material in method two, and "ICE-steel section UK open section" $\mathrm{ECF}$ with a value of $2.45 \mathrm{kgCO} 2 \mathrm{e} / \mathrm{kg}$ was assigned in method three. For aluminium framing of the curtain walls and windows, "ICE-aluminium general EU including transport" was assigned in method two with "ICE-aluminium extruded profile EU including trans- 
port" assigned in method three, with their values respectively being $6.669 \mathrm{kgCO} 2 \mathrm{e} / \mathrm{kg}$ and $1.706 \mathrm{kgCO} 2 \mathrm{e} / \mathrm{kg}$. The "Insulation" ECF with a value of $1.865 \mathrm{kgCO} 2 \mathrm{e} / \mathrm{kg}$ was assigned to all insulation layers of materials, including the internal layers of the roof and wall elements in method one. In methods two and three, "ICE-insulation" ECF with a value of $1.44 \mathrm{kgCO} 2 \mathrm{e} / \mathrm{kg}$ was assigned to the insulation's layers in the roof and internal walls. This ECF is also assigned to the insulation materials for the external walls in method two. While in method three, the ECF value for "Glass wool" derived from a publication by EU UTS in 2012 [22] equal to $0.74 \mathrm{kgCO} e / \mathrm{kg}$ was assigned. For the Polymethyl Methacrylate (PMMA) material layer in the external walls, "Plastics: average plastics" ECF was assigned in method one, and in method two, "ICE-Rubber" was assigned. In method three, the ECF for PMMA was derived from a dataset published by the University of Melbourne in 2019 [23] equal to $15.4 \mathrm{kgCO} e / \mathrm{kg}$. For plasterboard elements, the ECF for "Plasterboard" was assigned in method one, and "ICE-plasterboard" was assigned in methods two and three, with their values respectively being $0.12 \mathrm{kgCO} 2 \mathrm{e} / \mathrm{kg}$ and $0.39 \mathrm{kgCO} 2 \mathrm{e} / \mathrm{kg}$. For brick wall elements, the ECF for "Brick" was assigned in method one and "ICE-generic brick" was assigned in methods two and three. With their values respectively being $0.245 \mathrm{kgCO} 2 \mathrm{e} / \mathrm{kg}$ and $0.213 \mathrm{kgCO} 2 \mathrm{e} / \mathrm{kg}$. For glass elements, the ECF for "Glass" was assigned in method one, "ICE-glass general" was assigned in method two, and "ICE-glass toughened $3 \mathrm{~mm}^{\prime \prime}$ was assigned in method three, with their values respectively being $0.895 \mathrm{kgCO} 2 \mathrm{e} / \mathrm{kg}, 1.437 \mathrm{kgCO} 2 \mathrm{e} / \mathrm{kg}$, and $1.667 \mathrm{kgCO} 2 \mathrm{e} / \mathrm{kg}$ (Table 6).

Table 6. Assigned embodied carbon factor for each material of the standard supermarket design.

\begin{tabular}{|c|c|c|c|}
\hline Material Name & Method One ECF Source & Method Two ECF Source & Method Three ECF Source \\
\hline $\begin{array}{c}\text { Concrete, Cast-in-Place-C15 } \\
\text { Default Mass Floor } \\
\text { Concrete-Cast-in-Place Concrete } \\
\text { Concrete, Cast In Situ } \\
\text { Concrete }\end{array}$ & Concrete & ICE-Concrete, General & ICE-Concrete, General \\
\hline Concrete-Precast Concrete- $35 \mathrm{MPa}$ & Concrete & ICE-precast AAC block & ICE-Concrete 35/45 \\
\hline Steel & Metals & ICE-steel pipe & ICE-steel pipe \\
\hline $\begin{array}{l}\text { QuadCoreTrapezoidalRoofPanel_ } \\
\text { KS1000RW_ExternalWeatherSheet }\end{array}$ & Metals & ICE-Aluminium sheet EU & $\begin{array}{l}\text { ICE-Aluminium sheet EU inc } \\
\text { transport }\end{array}$ \\
\hline $\begin{array}{l}\text { QuadCoreTrapezoidalRoofPanel_ } \\
\text { KS1000RW_Internal Liner Sheet }\end{array}$ & Metals & ICE-Steel & ICE-Steel, organic coated sheet \\
\hline Default Roof-metal single skin & Metals & ICE-Steel & ICE-Steel, organic coated sheet \\
\hline Metal-Steel 50-355 & Metals & ICE-Steel section & $\begin{array}{l}\text { ICE-steel section UK open } \\
\text { sections }\end{array}$ \\
\hline Metal-Steel-S275 & Metals & ICE-Steel section & $\begin{array}{l}\text { ICE-steel section UK open } \\
\text { sections }\end{array}$ \\
\hline $\begin{array}{l}\text { Prepainted_Steel- } \\
\text { ArcelorMittal_Construction_HAIRULTRA- } \\
\text { 35-CORAL-4309-[RAL_3000] }\end{array}$ & Metals & ICE-Steel & ICE-Steel, organic coated sheet \\
\hline Cladding, Vertical Ribbed & Metals & $\begin{array}{l}\text { ICE-aluminium sheet general } \\
\text { EU including imports }\end{array}$ & $\begin{array}{l}\text { ICE-aluminium sheet general EU } \\
\text { including imports }\end{array}$ \\
\hline Metal Stud Layer & Metals & ICE-Steel & ICE-Steel \\
\hline Aluminium & Metals & $\begin{array}{l}\text { ICE-aluminium general EU } \\
\text { including transport }\end{array}$ & $\begin{array}{l}\text { ICE-aluminium extruded profile } \\
\text { EU including transport }\end{array}$ \\
\hline PIRFoamBoard_PIRInsulation & Insulation & ICE-Insulation & ICE-Insulation \\
\hline Default Roof-Generic insulation $125 \mathrm{~mm}$ & Insulation & ICE-Insulation & ICE-Insulation \\
\hline ArcelorMittal-Mineral Wool & Insulation & ICE-Insulation & ec.europa.eu-glass wool \\
\hline Insulation/Support Frame & Insulation & ICE-Insulation & ec.europa.eu-glass wool \\
\hline Rock Wool & Insulation & ICE-Insulation & ICE-Insulation \\
\hline Default Wall & Plasterboard & ICE-plasterboard & ICE-plasterboard \\
\hline Gypsum Wall Board & Plasterboard & ICE-plasterboard & ICE-plasterboard \\
\hline
\end{tabular}


Table 6. Cont

\begin{tabular}{cccc}
\hline Material Name & Method One ECF Source & Method Two ECF Source & Method Three ECF Source \\
\hline Plaster & Plasterboard & ICE-plasterboard & ICE-plasterboard \\
\hline Brick, Common & Bricks & ICE-generic brick & ICE-generic brick \\
\hline $\begin{array}{c}\text { Prepainted_Steel- } \\
\text { ArcelorMittalConstruction INTÉRIEUR } \\
\text { 12 WHITE 912 }\end{array}$ & Plastics: average plastics & ICE-Rubber & PMMA.pdf \\
\hline Paint-White Lining & Average construction & ICE-paint & ICE-paint \\
\hline
\end{tabular}

\section{Results and Discussion}

The results presented in Table 7 and Figure 7 show the significant effect the choice of ECF database can have on an LCA. While this study was conducted within the A1-A3 boundary, some ECFs such as the ECFs for aluminium sheets within the ICE database were defined by production location, either EU or international. If the material used by a project is produced within the same country, the use of this ECF could result in over calculation of the embodied carbon. In this scenario, the existence of an EPD published by the manufacturer would provide more accurate data. The transport emissions could then be calculated based on the projects site location.

For materials such as concrete used in foundations, the difference between using different databases is not as significant as the choice within one database. It can be assumed that this is due to the common use of concrete and the high volume of data available regarding this material [24]. The mitigation strategy for reduction of the embodied carbon in concrete is the replacement of cement with material with lower embodied carbon such as Pulverised Fuel Ash (PFA) while maintaining the desired strength. As presented by Gibbons and Orr in the 2020 istructe manual [8], this is an attainable undertaking. The ICE database provides several ECF for different concrete mixtures, which provides the data necessary for an EC reduction strategy. The most noticeable difference can be seen in the metal materials. When a project such as the model observed in this study is composed of a steel structure, this difference can be immense. Table 8 shows that this caused a 200-tonne $\mathrm{CO} 2 \mathrm{e}$ difference between the first and third methods. The first ECF database assigned a value of $4.769 \mathrm{kgCO} 2 \mathrm{e} / \mathrm{kg}$ to all metal materials, while the second database showed that the ECF for different metals could range between $1.706 \mathrm{kgCO} 2 \mathrm{e} / \mathrm{kg}$ and $6.669 \mathrm{kgCO} 2 \mathrm{e} / \mathrm{kg}$.

Furthermore, these values are only for virgin metal materials. As with cement, the first mitigation strategy for reducing embodied carbon in metal is the partial or total use of recycled metal. Additionally, it does not take into account the material production method with materials produced using a basic oxygen furnace (BOF) have a much higher embodied carbon, compared to the materials produced using an electric arc furnace (EAF) [8]. Another ECF that caused a noticeable difference is the ECF choice for the PMMA material. For this material, the necessity for detail originated from manufacturer-provided BIM building material in the model. Therefore, a more general approach was taken in the ECF assignment in methods one and two. For this building element which is the main element used in the building envelope, manufacturer provided EPD would be preferable.

The total embodied carbons presented in Table 8 shows that the use of more general ECF for embodied carbon calculations result in overcalculation. These results show that using the more detailed ECF compared to the more general database resulted in a $35.2 \%$ reduction of embodied carbon. The lack of a primary or nationalised database resulted in additional labour in performing an LCA. This point is a significant deterrent for the undertaking of an LCA as part of the design and construction process. Additionally, when embodied carbon calculations are carried during the design and construction process, it provides a baseline for the carbon reduction of that project or future projects. The overcalculation of embodied carbon resulting from a generalised approach could hinder reduction efforts by presenting the perception that a higher carbon reduction is possible. 
Furthermore, a detailed approach would require the sourcing of ECFs from several sources, reducing the reliability in the comparison stage of the EC reduction effort.

Table 7. The calculated embodied carbon for each material of the standard supermarket design.

\begin{tabular}{|c|c|c|c|}
\hline & Method One EC (kgCO2e) & Method Two EC (kgCO2e) & $\begin{array}{l}\text { Method Three EC } \\
(\text { kgCO2e) }\end{array}$ \\
\hline $\begin{array}{l}\text { Concrete, Cast-in-Place-C15 } \\
\text { Default Mass Floor } \\
\text { Concrete-Cast-in-Place Concrete } \\
\text { Concrete, Cast In Situ } \\
\text { Concrete }\end{array}$ & $16,534.20 \mathrm{kgCO} 2 \mathrm{e}$ & $12,682.80 \mathrm{kgCO} 2 \mathrm{e}$ & $12,682.80 \mathrm{kgCO} 2 \mathrm{e}$ \\
\hline Concrete, Cast-in-Place-C15 & $1978.1 \mathrm{kgCO} 2 \mathrm{e}$ & $3581.4 \mathrm{kgCO} 2 \mathrm{e}$ & $2058.0 \mathrm{kgCO} 2 \mathrm{e}$ \\
\hline Default Mass Floor & $160.5 \mathrm{kgCO} 2 \mathrm{e}$ & $123.1 \mathrm{kgCO} 2 \mathrm{e}$ & $123.1 \mathrm{kgCO} 2 \mathrm{e}$ \\
\hline Concrete-Cast-in-Place Concrete & $13,078.8 \mathrm{kgCO} 2 \mathrm{e}$ & $10,032.3 \mathrm{kgCO} 2 \mathrm{e}$ & $10,032.3 \mathrm{kgCO} 2 \mathrm{e}$ \\
\hline Concrete, Cast In Situ & $0.6 \mathrm{kgCO} 2 \mathrm{e}$ & $0.5 \mathrm{kgCO} 2 \mathrm{e}$ & $0.5 \mathrm{kgCO} 2 \mathrm{e}$ \\
\hline Concrete & $1316.2 \mathrm{kgCO} 2 \mathrm{e}$ & $1009.6 \mathrm{kgCO} 2 \mathrm{e}$ & $1009.6 \mathrm{kgCO} 2 \mathrm{e}$ \\
\hline Concrete-Precast Concrete- $35 \mathrm{MPa}$ & $1724.2 \mathrm{kgCO} 2 \mathrm{e}$ & $2276.7 \mathrm{kgCO} 2 \mathrm{e}$ & $3581.4 \mathrm{kgCO} 2 \mathrm{e}$ \\
\hline Steel & $21,597.1 \mathrm{kgCO} 2 \mathrm{e}$ & $13,676.8 \mathrm{kgCO} 2 \mathrm{e}$ & $13,676.8 \mathrm{kgCO} 2 \mathrm{e}$ \\
\hline $\begin{array}{l}\text { QuadCore_Trapezoidal_Roof_Panel_ } \\
\text { KS1000RW_ExternalWeatherSheet }\end{array}$ & $94,044.7 \mathrm{kgCO} 2 \mathrm{e}$ & $129,760.3 \mathrm{kgCO} 2 \mathrm{e}$ & $64,892.0 \mathrm{kgCO} 2 \mathrm{e}$ \\
\hline $\begin{array}{l}\text { QuadCore_Trapezoidal_Roof_Panel_ } \\
\text { KS1000RW_Internal_LinerSheet }\end{array}$ & $140,531.9 \mathrm{kgCO} 2 \mathrm{e}$ & $87,521.1 \mathrm{kgCO} 2 \mathrm{e}$ & $90,173.3 \mathrm{kgCO} 2 \mathrm{e}$ \\
\hline Default Roof-metal single skin & $208.8 \mathrm{kgCO} 2 \mathrm{e}$ & $130.1 \mathrm{kgCO} 2 \mathrm{e}$ & $134.0 \mathrm{kgCO} 2 \mathrm{e}$ \\
\hline Metal-Steel 50-355 & $100,064.7302 \mathrm{kgCO} 2 \mathrm{e}$ & $32,523.3 \mathrm{kgCO} 2 \mathrm{e}$ & $51,599.0 \mathrm{kgCO} 2 \mathrm{e}$ \\
\hline Metal-Steel-S275 & $301,353.4338 \mathrm{kgCO} 2 \mathrm{e}$ & $98,961.3 \mathrm{kgCO} 2 \mathrm{e}$ & $153,575.3 \mathrm{kgCO} 2 \mathrm{e}$ \\
\hline $\begin{array}{l}\text { PrepaintedSteel_ArcelorMittal_- } \\
\text { Construction_HAIRULTRA-35- } \\
\text { CORAL }\end{array}$ & $131,833.3 \mathrm{kgCO} 2 \mathrm{e}$ & $82,103.8 \mathrm{kgCO} 2 \mathrm{e}$ & $84,591.8 \mathrm{kgCO} 2 \mathrm{e}$ \\
\hline Cladding, Vertical Ribbed & $86,433.70 \mathrm{kgCO} 2 \mathrm{e}$ & $41,616.40 \mathrm{kgCO} 2 \mathrm{e}$ & $41,616.40 \mathrm{kgCO} 2 \mathrm{e}$ \\
\hline Metal Stud Layer & $808,949.3 \mathrm{kgCO} 2 \mathrm{e}$ & $503,801.6 \mathrm{kgCO} 2 \mathrm{e}$ & $503,801.6 \mathrm{kgCO} 2 \mathrm{e}$ \\
\hline Aluminium & $12,771.8 \mathrm{kgCO} 2 \mathrm{e}$ & $17,859.7 \mathrm{kgCO} 2 \mathrm{e}$ & $4569.7 \mathrm{kgCO} 2 \mathrm{e}$ \\
\hline PIRFoamBoard_PIRInsulation & $24,178.6 \mathrm{kgCO} 2 \mathrm{e}$ & $18,670.7 \mathrm{kgCO} 2 \mathrm{e}$ & $18,670.7 \mathrm{kgCO} 2 \mathrm{e}$ \\
\hline $\begin{array}{l}\text { Default Roof-Generic insulation } \\
125 \mathrm{~mm}\end{array}$ & $13.2 \mathrm{kgCO} 2 \mathrm{e}$ & $678.3 \mathrm{kgCO} 2 \mathrm{e}$ & $678.3 \mathrm{kgCO} 2 \mathrm{e}$ \\
\hline ArcelorMittal-Mineral Wool & $455,062.215 \mathrm{kgCO} 2 \mathrm{e}$ & $351,399.4 \mathrm{kgCO} 2 \mathrm{e}$ & $180,580.2 \mathrm{kgCO} 2 \mathrm{e}$ \\
\hline Insulation/Support Frame & $53.10 \mathrm{kgCO} 2 \mathrm{e}$ & $41.00 \mathrm{kgCO} 2 \mathrm{e}$ & $21.10 \mathrm{kgCO} 2 \mathrm{e}$ \\
\hline Rock Wool & $61,589.2 \mathrm{kgCO} 2 \mathrm{e}$ & $47,559.2 \mathrm{kgCO} 2 \mathrm{e}$ & $47,559.2 \mathrm{kgCO} 2 \mathrm{e}$ \\
\hline Default Wall & $92.3 \mathrm{kgCO} 2 \mathrm{e}$ & $299.6 \mathrm{kgCO} 2 \mathrm{e}$ & $299.6 \mathrm{kgCO} 2 \mathrm{e}$ \\
\hline Gypsum Wall Board & $4343.3 \mathrm{kgCO} 2 \mathrm{e}$ & $14,103.8 \mathrm{kgCO} 2 \mathrm{e}$ & $14,103.8 \mathrm{kgCO} 2 \mathrm{e}$ \\
\hline Plaster & $536.5 \mathrm{kgCO} 2 \mathrm{e}$ & $1742.3 \mathrm{kgCO} 2 \mathrm{e}$ & $1742.3 \mathrm{kgCO} 2 \mathrm{e}$ \\
\hline Brick, Common & $6348.4 \mathrm{kgCO} 2 \mathrm{e}$ & $5523.7 \mathrm{kgCO} 2 \mathrm{e}$ & $5523.7 \mathrm{kgCO} 2 \mathrm{e}$ \\
\hline $\begin{array}{l}\text { PrepaintedSteel_ArcelorMittal_ } \\
\text { Construction_INTÉRIEUR-12- } \\
\text { WHITE }\end{array}$ & $10,739.4 \mathrm{kgCO} 2 \mathrm{e}$ & $9158.4 \mathrm{kgCO} 2 \mathrm{e}$ & $49,487.2 \mathrm{kgCO} 2 \mathrm{e}$ \\
\hline Paint-White Lining & $0.0 \mathrm{kgCO} 2 \mathrm{e}$ & $1.3 \mathrm{kgCO} 2 \mathrm{e}$ & $1.3 \mathrm{kgCO} 2 \mathrm{e}$ \\
\hline Total & 2293.84 tonneCO2e & 1487.76 tonneCO2e & 1359.70 tonneCO2e \\
\hline
\end{tabular}




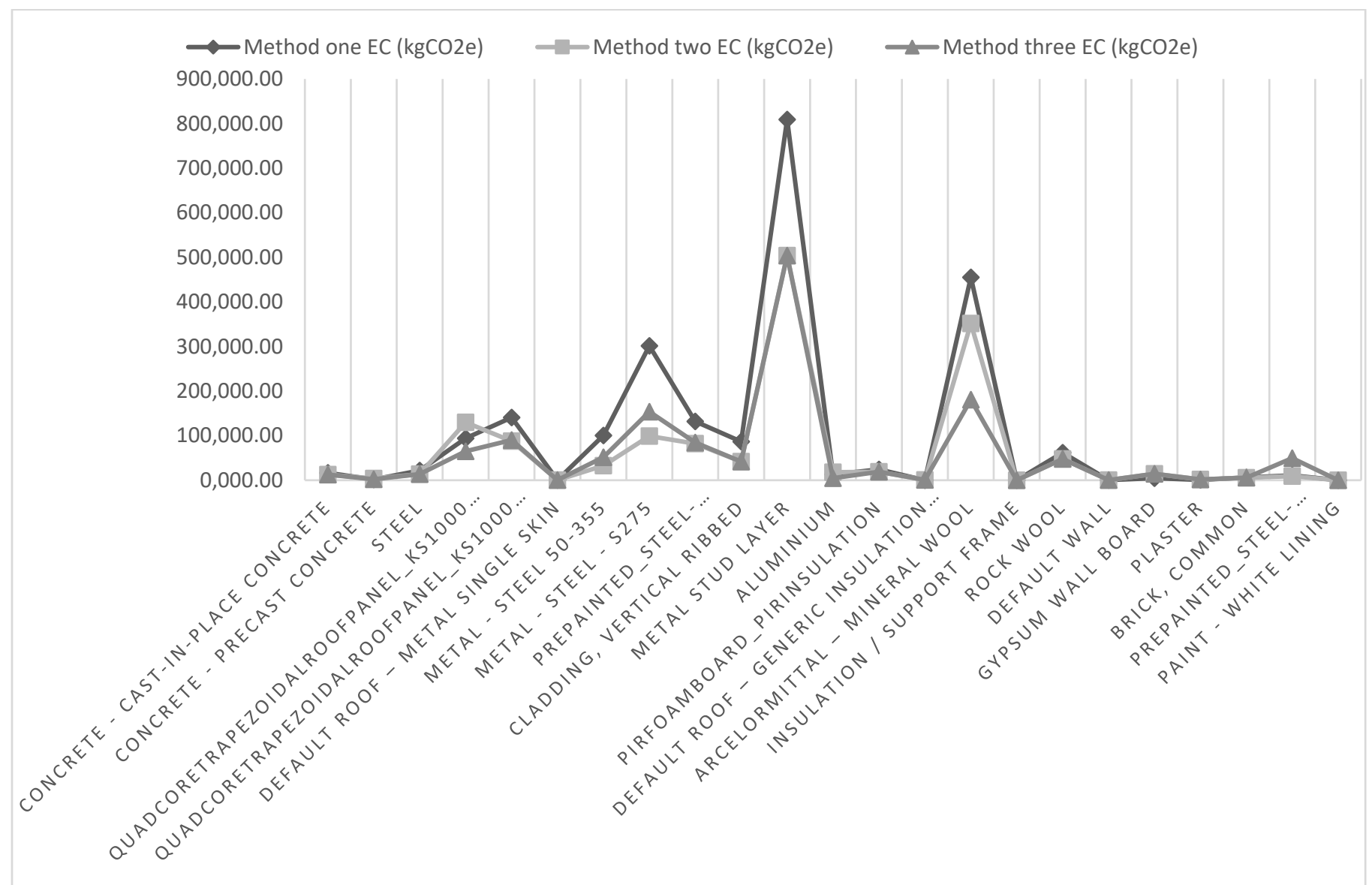

Figure 7. Calculated embodied carbon for each material of the standard supermarket design.

Table 8. The total embodied carbon for each structural element of the standard supermarket design.

\begin{tabular}{ccccc}
\hline Category & $\begin{array}{c}\text { Material: } \\
\text { Weight (tonne) }\end{array}$ & $\begin{array}{c}\text { Method One EC } \\
\text { (tonneCO2e) }\end{array}$ & $\begin{array}{c}\text { Method Two EC } \\
\text { (tonneCO2e) }\end{array}$ & $\begin{array}{c}\text { Method Three } \\
\text { EC (tonneCO2e) }\end{array}$ \\
\hline $\begin{array}{c}\text { Curtain Panels } \\
\text { Floors }\end{array}$ & 169.11 & 331.94 & 252.72 & 148.34 \\
$\quad \begin{array}{c}\text { Roofs } \\
\text { Structural }\end{array}$ & 14.67 & 1.98 & 1.52 & 1.52 \\
Columns & 62.37 & 258.98 & 236.76 & 174.55 \\
Structural & 20.98 & 100.06 & 32.52 & 51.60 \\
Foundations & 98.22 & 13.24 & 10.16 & 10.16 \\
Structural & & & & 157.35 \\
Framing & 64.73 & 308.69 & 101.35 & 785.58 \\
$\quad$ Walls & 416.48 & 1237.08 & 807.92 & 18.38 \\
Windows & 10.96 & 20.18 & 29.77 & 13.68 \\
Mechanical & 4.53 & 21.60 & 13.68 & 1359.70 \\
\hline TOTAL & 862.079 & 2293.84 & 1487.76 &
\end{tabular}

\section{Conclusions and Future Work}

Reducing the embodied carbon of buildings as a significant contributor to GHG emissions is a vital task that must be undertaken to reduce anthropogenic emissions and mitigate climate change. Performing Life Cycle Assessments is a vital step in the goal to reduce GHG emissions. The lack of a concrete approach in performing an LCA deters the undertaking of an LCA as part of the design and construction process. This study was 
conducted to observe the role of embodied carbon factor databases in the accuracy of an LCA and the adverse outcomes of the lack of an official national ECF database.

This study calculated the embodied carbon of a standard Lidl supermarket using a general database and a more detailed database, as well as choosing ECFs to be the closest to materials details. This study showed that using a more detailed database compared to using a more general database could result in a $35.2 \%$ reduction of calculated embodied carbon. Meanwhile, assigning ECFs to be the most accurate to materials details reduces it by a further $5.5 \%$. The accuracy of this assessment is directly connected to the accuracy of the embodied carbon factors used in the process. If an LCA is carried out to reduce the embodied carbon of a design, the overcalculation that can occur as a result of a general approach or database can create an inflated starting point for reduction. It is necessary to establish the most accurate baseline for embodied carbon so that any carbon reduction attempts can be as effective as possible. The limit of specific databases also impedes further calculations for GHG reduction. If an LCA is carried out as part of the early design process, the ECF database would need to provide multiple alternatives. The consistent collection method within an ECF database would guarantee that the comparison of different designs would be viable. This study showed that while reliable databases for embodied carbon factors are available, the lack of an official national database can confuse the LCA process. This confusion results in additional necessary labour in performing an LCA, acting as a disincentive for carrying out an EC calculation.

The ICE database is currently the most reliable in the UK, most recently updated in 2019. However, the 7-year period between this update and the previous update in 2012 created the necessity to produce other databases. If manufacturer data is used to calculate and model a design, then the ECF must be sourced separately. A manufacturer-provided EPD would be preferable. However, as currently there are no regulations for manufacturers to produce EPDs, this method would be unlikely. These additional steps to perform an LCA create another obstacle preventing more action in this area. Requiring EPD publication by manufacturers and expanding and officialising ECF databases are necessary future steps in this field.

Author Contributions: Conceptualisation, A.B.-J., M.F., and A.M.; Research, 3D modelling, calculation and formal analysis, G.M.; Writing-original draft preparation and editing, G.M.; Writingreview and editing, and supervision, A.B.-J., M.F., and A.M. All authors have read and agreed to the published version of the manuscript.

Funding: This research received no external funding.

Data Availability Statement: All data generated from this study is available within the text of this manuscript.

Conflicts of Interest: The authors declare no conflict of interest.

\section{References}

1. UNEP. 2019 Global Status Report for Buildings and Construction Sector. 11 December 2019. Available online: https:// www.unenvironment.org/resources/publication/2019-global-status-report-buildings-and-construction-sector (accessed on 1 December 2020).

2. Pomponi, F.; de Wolf, C.; Moncaster, A. Embodied Carbon in Buildings, 1st ed.; Springer: Cham, Switzerland, 2018.

3. Roberts, M.; Allen, S.; Coley, D. Life cycle assessment in the building design process-A systematic Literature review. Build. Environ. 2020, 185, 107274. [CrossRef]

4. Kotaji, S.; Edwards, S.; Schuurmans, A. Life Cycle Assessment in Building and Construction: A State-of-the-Art Report; SETAC Press: Brussels, Belgium, 2003.

5. Nassen, J.; Holmberg, J.; Wadeskok, A.; Nyman, M. Direct and indirect energy use and carbon emissions in the production phase of buildings: An input-output analysis. Energy 2007, 32, 1593-1602. [CrossRef]

6. Ibn-Mohammed, T.; Greenougha, R.; Taylor, S.; Ozawa-Meidaa, L.; Acquayec, A. Operational vs embodied emissions in buildings-A review of current trends. Energy Build. 2013, 66, 232-245. [CrossRef]

7. Negm, A.M.; AbdelMontaleb, A.; Bady, M.F. Estimating Carbon Emissions from Industrial Process by Using Life Cycle Assessment Too; Egypt-Japan University of Science and Technology: Alexandria, Egypt, 2013.

8. Gibbons, O.P.; Orr, J.J. How to Calculate Embodied Carbon (Istructe); The Institution of Structural Engineers: London, UK, 2020. 
9. UK Department of Energy \& Climate Change. "Gov.uk". 5 October 2020. Available online: https://www.gov.uk/government/ publications / greenhouse-gas-reporting-conversion-factors-2020 (accessed on 4 May 2021).

10. Yohanis, Y.; Norton, B. Life Cycle operational and embodied energy for a generic single-story office building in the UK. Energy 2002, 22, 77-92. [CrossRef]

11. Butcher, K. Embodied Carbon in Building Services-A Calculation Methodology-CIBSE TM 65; Chartered Institution of Building Services Engineers: London, UK, 2021.

12. ISO. Environmental Management_Life Cycle Assessment_-Requirements and Guidelines. ISO 14044; International Organization for Standardization: Geneva, Switzerland, 2006.

13. ISO. ISO 14044:2006/AMD 2:2020—Environmental Management_Life Cycle Assessment_Requirements and Guidelines—Amendment 2; International Organization for Standardization: Geneva, Switzerland, 2020.

14. ISO. ISO 14040:1997 Environmental Management_Life Cycle Assessment_Principles and Framework; International Organization for Standardization: Geneva, Switzerland, 1997.

15. Hsu, S.; Ochsendorf, J.; Veneziano, D. Life Cycle Assessment of Materials and Construction in Commercial Structures: Variability and Limitations. Ph.D. Thesis, Massachusetts Institute of Technology, Cambridge, MA, USA, 2010.

16. Omar, W.M.S.W.; Doh, J.H.; Panuwatwanish, K.; Miller, D. Assessment of the embodied carbon in precast concrete wall panels using a hybrid life cycle assessment approach in Malaysia. Sustain. Cities Soc. 2014, 10, 101-111. [CrossRef]

17. Richardson, S. Embodied Carbon Assessment and Decision Making Under Uncertainty: Case Studies of UK Supermarket Construction; University of Reading: Reading, UK, 2017.

18. Anderson, J. Constructionlca's Guide to EPD—Infogram, Charts \& Inforgraphics. 2017. Available online: http://infogr.am/4721 6efb-7256-4a5e-acc3-04ce046cbdf8 (accessed on 3 May 2021).

19. Anderson, J. Greenspec. 2020. Available online: https://www.greenspec.co.uk/building-design/embodied-energy/ (accessed on 22 April 2021).

20. Jones, G.H.C. Embodied Carbon-The ICE Database. November 2019. Available online: https:// circularecology.com/embodiedcarbon-footprint-database.html (accessed on 28 January 2021).

21. Rogers, P.; Murray, J.; Cain, M.; Hussey, L.; James, B.; Lane, B.; Robertson, C.; Simpson, M. Specification for Sheet metal Ductwork: Low, Medium and High. Pressure/Velocity Air Systems; Publications BESA Publications: Penrith, Cumbri, 2016.

22. Ecofys, Fraunhofer Institute for Systems and Innovation Research; Öko-Institut. Methodology for the Free Allocation of Emission Allowances in the EU-Sector Report for the Mineral. Wool Industry; European Commission: Brussels, Belgium, 2012.

23. Crawford, R.; Stephan, A.; Prideaux, F. EPiC Database_Polymethyl Methacrylate (PMMA); University of Melbourne: Melbourne, Australia, 2019.

24. Nazari, A. Handbook of Low Carbon Concrete; Butterworth-Heinemann: Amsterdam, The Netherlands, 2016. 